\title{
The abundance of a transfer RNA-derived RNA fragment small RNA subpopulation is enriched in cauda spermatozoa
}

\author{
Simone J. Stanger', Ilana R. Bernstein ', Amanda L. Anderson', Kate Hutcheon², Matthew D. Dun ${ }^{3,4}$, \\ Andrew L. Eamens ${ }^{1 \dagger}$ and Brett Nixon ${ }^{1 * \dagger}$ (ID
}

\begin{abstract}
The small RNA (sRNA) landscape of mammalian spermatozoa is considerably altered as these gametic cells migrate through the segment specific microenvironments of the epididymis. More specifically, the microRNA (miRNA) species of sRNA dominates the sRNA landscape of spermatozoa of the proximal caput segment of the epididymis. However, in sperm cells sourced from the distal cauda epididymal segment, the transfer RNA (tRNA)-derived RNA fragment (tRF) sRNA species is the most abundant. Here we show that the $5^{\prime}$ halves of fifteen mature tRNAs were used as processing substrates for the production of a specific subpopulation of tRF sRNAs, 30 to 33 nucleotides (30-33-nt) in length. A quantitative reverse transcriptase polymerase chain reaction (RT-qPCR) approach was used to experimentally validate the sRNA sequencing identified trend of enriched abundance of this specific 30-33-nt tRF subpopulation in cauda spermatozoa. The length, and exclusive alignment of the cauda spermatozoa enriched tRF subpopulation to the $5^{\prime}$ half of each processed tRNA precursor, identified ANGIOGENIN (ANG) as the endonuclease likely responsible for tRF production in the mouse epididymis: a prediction confirmed via immunoblotting assessment of ANG abundance in spermatozoa sourced from the caput, corpus and cauda epididymal segments. When taken together with our previous profiling of miRNA and Piwi-interacting RNA (piRNA) sRNA abundance in spermatozoa sourced from the three segments of physiologically normal mouse epididymides, the tRF profile reported here adds greater depth of coverage to the global sRNA landscape of the mouse epididymis; a roadmap constructed to assist with the future molecular characterization of sRNA-directed responses to a wide range of imposed environmental stressors.
\end{abstract}

Keywords: Mouse epididymis, Caput, Corpus and cauda epididymis, Spermatozoa, sRNA abundance, tRF subpopulation enrichment, sRNA sequencing, RT-qPCR, ANGIOGENIN, Immunoblotting

\section{Background}

In mammals, spermatozoa must undergo sequential phases of mitosis, meiosis and cytological differentiation, before they are rendered morphologically mature [1]. Despite the level of specialization achieved during these combined phases of spermatogenesis, the spermatozoa

\footnotetext{
* Correspondence: brett.nixon@newcastle.edu.au

${ }^{\dagger}$ Andrew L. Eamens and Brett Nixon contributed equally to this work.

'Priority Research Centre for Reproduction Science, School of Environmental and Life Sciences, The University of Newcastle, Callaghan, New South Wales 2308, Australia

Full list of author information is available at the end of the article
}

generated by the germinal epithelium of the testes are functionally immature upon release into the epididymal tubule. Indeed, it is not until their prolonged migration through the caput, corpus and cauda segment-specific microenvironments of the epididymis, that sperm gain forward progressive motility and capacitation competency as well as acquiring an affinity for the cumulusoocyte complex [2]. As part of their epididymal maturation, sperm undergo a conspicuous morphological transformation via the shedding of the remnants of their cytoplasm held within the cytoplasmic droplet, and the

(C) The Author(s). 2020 Open Access This article is licensed under a Creative Commons Attribution 4.0 International License, which permits use, sharing, adaptation, distribution and reproduction in any medium or format, as long as you give

appropriate credit to the original author(s) and the source, provide a link to the Creative Commons licence, and indicate if changes were made. The images or other third party material in this article are included in the article's Creative Commons licence, unless indicated otherwise in a credit line to the material. If material is not included in the article's Creative Commons licence and your intended use is not permitted by statutory regulation or exceeds the permitted use, you will need to obtain permission directly from the copyright holder. To view a copy of this licence, visit http://creativecommons.org/licenses/by/4.0/. 
extensive remodelling of their haploid genome [3-5]. The drastically altered morphology of mature spermatozoa renders these cells silent, both at the transcriptional and translational level. Therefore, their functional maturation in the epididymis must be driven extrinsically: a process made possible by the secretive and absorptive activity of the epithelium that lines the lumen of the tract [6].

Historical research has focused heavily on the characterization of the complex interplay between the secretory and absorptive activity of the soma of each epididymal segment, and the maturing spermatozoa descending the tract [7]. More specifically, much of this research attention was aimed at documenting the complex segment-specific protein complement secreted into the epididymis lumen in an attempt to identify the molecules that direct the functional maturation of spermatozoa $[8,9]$. Via apocrine secretion, a heterogenous population of membrane enclosed extracellular vesicles, collectively termed epididymosomes, are now viewed as a central component of epididymal soma-spermatozoa intercellular communication [10-15]. As demonstrated for exosome populations generated by various other somatic cell types, epididymosomes are likely capable of mediating the transfer of a rich and functionally diverse RNA and protein cargo to their recipient cells [16-22]. Considering that the recipient spermatozoa are rendered transcriptionally and translationally silent prior to entry into the tract, the communicative role occupied by epididymosomes takes on further significance.

The more recent application of high throughput sequencing technologies to the male reproductive tract has repeatedly demonstrated that in addition to carrying an impressive repertoire of functionally diverse protein cargo, the soma, extracellular vesicles and spermatozoa of the mammalian epididymis, also harbor rich RNA payloads [16-20, 23]. Furthermore, the RNA profile of epididymal sperm is modified considerably as these cells descend the tract, a consequence believed to be the result of the considerable transcriptomic plasticity of the epithelium along the length of the tract with at least six transcriptionally distinct regions identified in the mouse model [24]. Such diversity in epididymal soma gene expression indicates that a multi-tiered regulatory network is at play to finetune the composition of the segment-bysegment transcriptome profile with neither androgens, nor other testis synthesized lumicrine factors believed sufficient to mediate such intricate gene expression coordination [25]. Small RNAs (sRNAs), short nonprotein-coding RNA molecules form a functionally diverse class of gene expression regulatory molecule, in both somatic and gametic cells. As such, sRNA-directed RNA silencing forms a prime candidate to add an additional layer of complexity to gene expression regulation in the epididymis [26]. Accordingly, the seminal work conducted by the Zhang [27-31], Sipilä [32], and Sullivan and Belleannee $[33,34]$ research teams, has provided elegant demonstration of such a role for gene expression regulation by distinct sRNA-directed RNA silencing mechanisms in the mammalian epididymis.

Due to their folding to form RNA secondary structures as part of their maturation [35-41], ribosomal (rRNA) and transfer RNA (tRNA) transcripts form suitable substrates for endonuclease catalyzed cleavage. To date, numerous sRNA species have been documented to accumulate in mammalian cells from the processing of RNA transcripts that adopt secondary structures, including the microRNA (miRNA), endogenous small-interfering RNA (endo-siRNA), anti-sense RNA (asRNA), interference RNA (iRNA), tRNA-derived RNA fragment (tRF), piwi-interacting RNA (piRNA), rRNA, small nuclear RNA (snRNA) and small nucleolar RNA (snoRNA) species of sRNA [14, 42-45]. Among these, and in the mammalian male reproductive tract, the miRNAs and piRNAs are the most thoroughly characterized due to the respective regulatory roles that these two sRNA species direct in the control of developmental gene expression and transposon activity suppression, respectively [17, 18, 20, 46-48]. However, due to the now readily apparent role that sRNAs play in directing transgenerational inheritance has come a flourish of recent research activity into uncovering the exact regulatory roles mediated by the other, less well characterized species of sRNA, such as the tRF species of small regulatory RNA (extensively reviewed in $[15,21]$ ).

Here, we report on our ongoing sRNA profiling of spermatozoa as these cells transit the mouse epididymis $[16,23,48-50]$ specifically focusing on the tRF species of sRNA, which undergoes a 2.7 -fold enrichment in abundance in cauda compared to caput spermatozoa. The tRF profile reported here adds greater depth of coverage to the global sRNA landscape of the mouse epididymis, and when taken together, our studies form a timely series of experimental analyses which facilitate the construction of an extensive roadmap of the sRNA landscape of the mouse epididymis: a roadmap that will allow for the future assessment of sRNA-directed gene expression regulatory responses to a wide range of imposed environmental stressors.

\section{Results}

The small RNA accumulation profile of mouse spermatozoa is dramatically altered during their epididymal transit

Assessment of the distribution of sRNA molecules 19-36nt in length across spermatozoa sourced from the proximal caput, to distal cauda segment of the mouse epididymis, revealed strikingly different abundance profiles (Fig. 1a). More specifically, at $18.6 \%$, sRNAs 22-nt in length dominated the accumulation profile of caput spermatozoa (Additional file 1: Table S1). This predominant peak was 


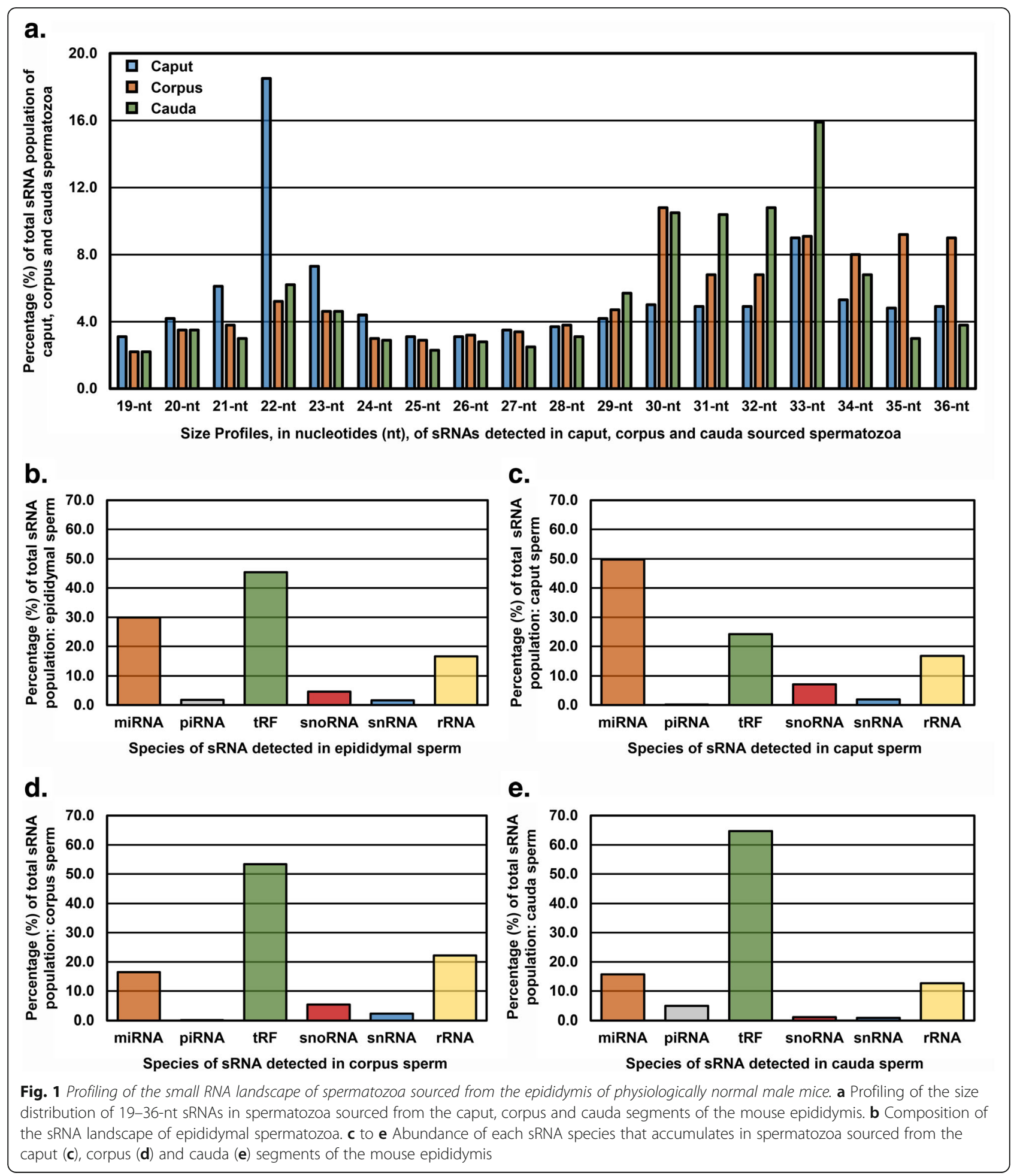

not observed in either the corpus or cauda spermatozoa profiles with 22-nt sRNA accumulation only representing 5.2 and $6.2 \%$ of total sRNA read counts, respectively (Fig. 1a; Additional file 1: Table S1). In spermatozoa sourced from the corpus segment of the mouse epididymis, the sRNA accumulation profile was evenly distributed across the 19-29-nt size window, however in comparison, 30-36nt sRNAs were determined to accumulate to a higher degree in corpus sperm cells (Fig. 1a; Additional file 1: Table S1). For spermatozoa sourced from the distal cauda segment of the mouse epididymis, further elevation to the abundance of 30-36-nt sRNAs formed a distinct 
accumulation peak (Fig. 1a), with sRNAs of this size range accounting for $47.6 \%$ of the total sRNA population of cauda sperm. In comparison, 30-36-nt sRNAs only represented 23.8 and $33.4 \%$ of the total $19-36$-nt sRNA populations of caput and corpus spermatozoa, respectively (Additional file 1: Table S1).

We have previously demonstrated that in spermatozoa sourced from the caput, corpus and cauda segments of the mouse epididymis, piRNA accumulation is enriched in cauda sperm [23]. More specifically, 348,445 sRNA reads were aligned to known mouse piRNAs in cauda spermatozoa (to represent $4.9 \%$ of total cauda sperm mapped sRNA reads) compared to only $18,742(0.2 \%$ of total reads) and 3991 reads ( $0.1 \%$ of total reads) mapping to mouse piRNA sRNAs in caput and corpus spermatozoa, respectively (Additional file 1: Table S2). Of the 348,445 reads mapped to piRNAs in cauda sperm, $60 \%$ ( 209,067 reads) were 29-31-nt in length, a sRNA size that indicated the requirement of PIWIL1 for their production [51-53]. The remaining piRNA sRNAs detected in cauda spermatozoa $(\sim 139,378$ reads) were slightly shorter in length at 26-28-nt, to suggest that this piRNA cohort represented products of the secondary piRNA production pathway mediated by PIWIL2 and PIWIL4 [51-53]. Therefore, considering that mouse piRNAs at 26-31-nt are slightly shorter in length, and only partially overlap with the accumulation peak observed in cauda spermatozoa at 30-33-nt by 2-nt, we next sought to determine which of the other sRNA species known to accumulate in mammalian spermatozoa this cauda sperm enriched sRNA size class belonged to.

Mapping of sRNA sequencing reads to each of the sRNA species known to accumulate in mammalian sperm, including the miRNA, piRNA, tRF, snoRNA, snRNA and rRNA species of sRNA, revealed that miRNAs and tRFs accumulated to the highest levels in epididymis sourced spermatozoa (Fig. 1b), to represent $29.9 \%(6,017,472$ reads $)$ and $45.4 \%(9,126,637$ reads $)$ of the 20,094,840 sRNA reads detected across caput, corpus and cauda sperm cells, respectively (Additional file 1: Table S2). Considering the documented peak in sRNA abundance at 22-nt in caput spermatozoa (Fig. 1a), it was unsurprising to subsequently determine that the miRNA species of sRNA was the most abundant in sperm cells sourced from the proximal segment of the mouse epididymis, representing $49.8 \%(4,125,107$ reads) of the 8,287,165 mapped sRNA reads (Fig. 1c; Additional file 1: Table S2). miRNA abundance was reduced in spermatozoa sourced from the two more distal epididymal segments to only represent $16.5 \%$ (Fig. 1d) and $15.7 \%$ (Fig. 1e) of the 4,653,242 and 7,154,433 reads mapped to known sRNAs in corpus and cauda sperm, respectively (Additional file 1: Table S2). Reduced miRNA abundance in corpus sperm was primarily accounted for by the increased accumulation of the tRF species of sRNA. Specifically, tRF sRNAs only represented $24.2 \%$ of the total mapped sRNA population of caput sperm (Fig. 1c), however the abundance of this sRNA species increased by 2.2 -fold to represent $53.4 \%$ of the total mapped sRNA population of corpus sperm (Fig. 1d; Additional file 1: Table S2). Increased tRF levels also accounted for the reduction to miRNA abundance in sperm cells sourced from the distal cauda segment of the epididymis (Fig. 1e). Namely, of the 7,154,433 sRNA reads mapped to known mouse sRNAs, $64.7 \%(4,631,726$ reads) were aligned to tRFs (Additional file 1: Table S2). Therefore, although we have previously shown that piRNA accumulation is enriched in cauda sperm [23], the profiling data presented in Fig. 1 clearly shows that the 3.2-fold reduced abundance of the miRNA species of sRNA in cauda (15.7\%) versus caput (49.8\%) spermatozoa, was largely accounted for by the reciprocal 2.7 -fold increase in the levels of the tRF species of sRNA in cauda spermatozoa (64.7\%), compared to the abundance (24.2\%) of this sRNA species in caput sperm cells (Additional file 1: Table S2).

\section{A specific subpopulation of transfer RNA-derived RNA fragment small RNAs is enriched in cauda spermatozoa} Profiling of the size distribution of the sRNA population of spermatozoa sourced from each segment of the mouse epididymis revealed that the accumulation of sRNA molecules 30-36-nt in length was enriched in corpus sperm cells (Fig. 1a). Figure 1a further shows that the abundance of 30-33-nt sRNAs was enriched to an even greater degree in spermatozoa sourced from the distal cauda segment of the mouse epididymis, and subsequently, the tRF species of sRNA was determined to account for the majority of the cauda spermatozoa sRNA landscape (Fig. 1e). Therefore, in order to further assess the degree of enrichment of tRF accumulation in spermatozoa, the read abundance of tRFs 27-35-nt in length was next determined for epididymal spermatozoa (Fig. 2).

In cauda sperm cells, a total of 4,631,726 reads mapped to 27-35-nt tRFs (Additional file 1: Table S3). In comparison, only 937,329 and 986,027 reads mapped to tRF sRNAs 27-35-nt in length in caput and corpus sperm, respectively (Additional file 1: Table S3). This represents a 4.9- and 4.7-fold enrichment in the abundance of 27-35-nt tRFs in sperm cells sourced from the distal cauda segment of the epididymis, compared to this size of tRF sRNA that accumulated in spermatozoa sourced from the two more proximal segments of the mouse epididymis (Fig. 2a; Additional file 1: Table S3). Furthermore, Fig. 2a also clearly shows the highly enriched abundance of 30-33-nt tRFs in cauda sperm cells compared to the abundance of tRF sRNAs of this size in spermatozoa sourced from either the caput or 


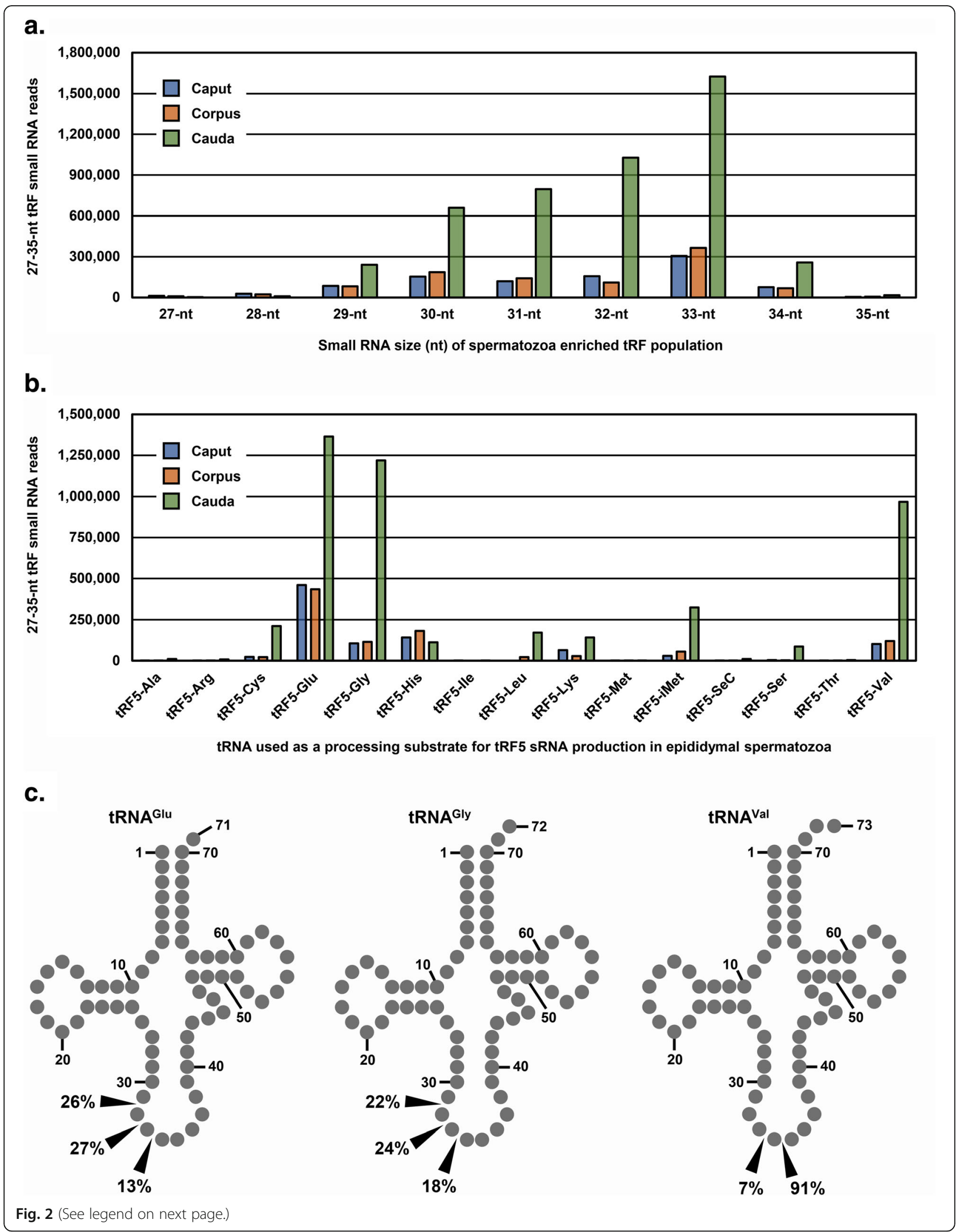


(See figure on previous page.)

Fig. 2 Composition of the transfer RNA-derived RNA fragment small RNA population of epididymal spermatozoa. a Profiling of the size distribution of 27-35-nt tRF sRNAs in spermatozoa sourced from the caput, corpus and cauda segments of the mouse epididymis. b Small RNA read numbers for the 15 tRF $5 s$ with enriched abundance in the cauda spermatozoa in sperm cells sourced from the caput, corpus and cauda epididymis. $\mathbf{c}$ Cleavage site preference for the tRNA ${ }^{\text {Glu }}$, tRNA ${ }^{\text {Gly }}$ and tRNA ${ }^{\text {Val }}$ substrates from which the three most abundant tRF5 sRNAs (tRF5 $5^{\text {Glu }}$, tRF5 ${ }^{\text {Gly }}$ and $\mathrm{tRF}^{\mathrm{Val}}$ ) in cauda spermatozoa were processed

corpus epididymal segment. More specifically, 4,105,965 reads were mapped to the tRF species of sRNA, 30-33nt in length, in cauda sperm (Additional file 1: Table S3). In comparison, only 734,168 and 800,197 reads were mapped to 30-33-nt tRFs in caput and corpus spermatozoa, respectively (Fig. 2a; Additional file 1: Table S3). In addition, tRF sRNAs 33-nt in length were determined to accumulate to the highest level in cauda sperm to total 1,624,413 reads. However, comparison of cauda sperm tRF abundance to either caput or corpus epididymis sourced sperm cells, revealed that the accumulation of 32-nt tRFs was enhanced to the greatest degree, with the abundance of 32-nt tRF sRNAs upregulated by 6.6and 9.3-fold in cauda spermatozoa (1,027,237 reads) compared to caput (156,118 reads) and corpus spermatozoa (110,061 reads), respectively (Fig. 2a).

Previous research conducted across a diverse range of; (1) species; (2) cell and/or tissue types, and; (3) experimental/environmental conditions has revealed that the tRF species of sRNA is composed of multiple, distinct, subpopulations [42, 43, 54-57]. Post transcription, processing, modification of specific ribonucleotides for secondary structure reinforcement, and the addition of a CCA trinucleotide at the $3^{\prime}$ terminus, each tRNA folds to form a highly conserved 'three leaf clover' structure composed of four structurally distinct arms, including the D-loop, anticodon arm, TYC arm and the variable loop [38, 42, 44, 58, 59]. Endonuclease catalyzed cleavage of any of the highly conserved structural features of a mature tRNA, or of the precursor transcript (pre-tRNA) intermediates of a tRNA, results in the production of a distinct tRF subpopulation. Namely; (1) the 5 ' series of tRF sRNAs (tRF5s) results from endonuclease-catalyzed processing of the D-loop of the tRNA molecule back to the $5^{\prime}$ terminus of the tRNA; (2) the $3^{\prime}$ tRF series (tRF3s) is generated via cleavage of the TYC arm through to the $3^{\prime}$ terminus of the molecule; (3) the U tRF3 series of tRF sRNAs (also referred to as the tRF-1 series) are produced via the cleavage of the 3 ' side of the pre-tRNA/tRNA, past the terminus of the transcript and to which a variable number of uracil $(\mathrm{U})$ residues are added, and; (4) internal tRFs (itRFs) are generated by a double cleavage event (cleavage sites are usually located in the D-loop and TYC arm of the processed pre-tRNA/ tRNA, respectively) of the pre-tRNA/tRNA across the anticodon arm of the molecule [44, 58, 59]. In addition to each of these tRF subpopulations, numerous mature
tRNA molecules are cleaved at, or immediately adjacent to, the anticodon trinucleotide of the anticodon arm. The sRNAs produced by the cleavage of the anticodon arm of a mature tRNA generates tRF molecules of a greater length (30-40-nt) than the other subpopulations of tRFs reported, and as such, are classed as 'tRNA halves' [31, 45, 59-61]. Alignment of the sRNA sequence of each cauda spermatozoa enriched tRF, revealed that this subpopulation exclusively aligned to the $5^{\prime}$ half of each mature tRNA molecule used as a substrate for tRF processing. This analysis further revealed highly specific cleavage of the anticodon arm, 2- to 3-nt immediately 5' (upstream) of the anticodon triplet of each processed mature tRNA. From herein, we therefore refer to each tRF that belonged to the cauda spermatozoa enriched tRF subpopulation (Fig. 2b; Additional file 1: Table S4), as a tRF5 sRNA, followed by the three-letter abbreviation of the tRNA from which the tRF5 SRNA was processed.

Assessment of the tRF5 landscape of caput, corpus and cauda spermatozoa, revealed that 15 tRNAs were used as processing substrates for tRF5 production (Fig. 2b; Additional file 1: Table S4). The sRNA, tRF5 $5^{\mathrm{Glu}}$, accumulated to the highest degree to total 2,258,567 reads, with 460,049, 434,536 and 1,363,982 reads detected in caput, corpus and cauda sourced sperm cells, respectively (Fig. 2b; Additional file 1: Table S4). Although considerably reduced in abundance compared to the tRF5 ${ }^{\mathrm{Glu}}$ sRNA at $1,439,663$ reads, tRF $^{\text {Gly }}$ was the second most abundant tRF5 sRNA across the caput (106,104 reads), corpus (114,735 reads) and cauda $(1,218,824$ reads) spermatozoa sequencing datasets. After the tRF5 ${ }^{\text {Glu }}$ and tRF5 $^{\text {Gly }}$ sRNAs, tRF5 ${ }^{\mathrm{Val}}$ was the third most abundant tRF5 sRNA detected in mouse spermatozoa $(1,189,651$ reads) sourced from the caput (102,593 reads), corpus (119,400 reads) and cauda (967,658 reads) epididymis (Additional file 1: Table S4). Taken together, the three most abundant tRF5 sRNAs detected in epididymal spermatozoa, including tRF5 ${ }^{\text {Glu }}$ (34.5\%), tRF5 ${ }^{\text {Gly }}$ (22.0\%) and $\mathrm{tRF}^{\mathrm{Val}}(18.1 \%)$, accounted for $74.6 \%(4,887,881$ of $6,555,082$ reads) of the total enriched tRF5 subpopulation (Fig. 2b; Additional File 1: Table S4). In addition to the three most abundant tRF sRNAs, Fig. 2b clearly shows that the accumulation of 11 other tRF5s (including $\mathrm{tRF}^{\mathrm{Ala}}$, $\mathrm{tRF} 5^{\mathrm{Arg}}, \mathrm{tRF}^{\mathrm{Cys}}, \mathrm{tRF} 5^{\mathrm{Ile}}, \mathrm{tRF} 5^{\mathrm{Leu}}, \mathrm{tRF} 5^{\mathrm{Lys}}$, $\mathrm{tRF}^{\mathrm{Met}}, \mathrm{tRF}^{\mathrm{iMet}}, \mathrm{tRF}^{\mathrm{SeC}}, \mathrm{tRF} 5^{\mathrm{Ser}}$ and $\mathrm{tRF} 5^{\mathrm{Thr}}$ ) was also enriched in cauda spermatozoa, compared to the abundance of each of these sRNAs in sperm cells sourced 
from either the caput or corpus epididymis. Therefore, of the 15 tRF5 sRNAs determined to belong to the cauda spermatozoa enriched tRF5 subpopulation, only the accumulation of the tRF5 ${ }^{\text {His }}$ sRNA was determined to be higher in caput (142,366 reads) and corpus (182,475 reads) spermatozoa than in cauda (112,043 reads) sperm cells (Additional file 1: Table S4).

The size of each cauda spermatozoa enriched tRF5 sRNA (Fig. 2a), together with the exclusive alignment of each tRF5 sRNA sequence to the $5^{\prime}$ half of the 15 tRNA molecules from which these sRNAs were processed (Fig. $2 b$ ), led us to next map the sRNA read sequences for the three most abundant tRF5s to their respective tRNAs (Fig. 2c). For the tRF5 $5^{\mathrm{Glu}}$ and tRF5 ${ }^{\mathrm{Gly}}$ sRNAs, three cleavage sites were mapped to the tRNA ${ }^{\text {Glu }}$ and tRNA ${ }^{\text {Gly }}$ molecules. More specifically, the 3 ' terminal nucleotide of 26 , 27 and $13 \%$ of $\mathrm{tRF} 5^{\mathrm{Glu}}$ sRNA sequencing reads aligned to the $31^{\text {st }}, 32^{\text {nd }}$ and $33^{\text {rd }}$ nt from the $5^{\prime}$ terminus of the tRNA $^{\text {Glu }}$ molecule to account for 587,227, 609,813 and 293,614 of the $2,258,567$ tRF5 $^{\text {Glu }}$ sequencing reads, respectively (Fig. 2c). Alignment of sRNA sequencing reads to the tRNA ${ }^{\text {Gly }}$ molecule revealed a highly similar result, that is; $22 \%$ (316,726 reads), $24 \%$ (345,519 reads) and $18 \%$ $\left(259,139\right.$ reads) of $\mathrm{tRF} 5^{\mathrm{Gly}}$ sRNA reads mapped to the $31^{\text {st }}$, $32^{\text {nd }}$ and $33^{\text {rd }}$ nt from the $5^{\prime}$ terminal nt of the tRNA ${ }^{\text {Gly }}$ molecule (Fig. 2c). Detection of tRF5s of the same length, and of a similar cleavage frequency from the tRNA ${ }^{\text {Glu }}$ and tRNA $^{\text {Gly }}$ molecules, indicated that in epididymal spermatozoa, these two tRNA substrates are processed at a similar degree of accuracy by the endonuclease responsible for tRF5 production. Mapping of $\mathrm{tRF} 5^{\mathrm{Val}}$ sequencing reads to the $\mathrm{tRNA}^{\mathrm{Val}}$ molecule indicated that $\mathrm{tRNA}^{\mathrm{Val}}$ formed a more suitable substrate for accurate endonucleasecatalyzed processing than did either tRNA ${ }^{\text {Glu }}$ or tRNA ${ }^{\text {Gly }}$ (Fig. 2c). Only two tRF5s, 33- and 34-nt in length, dominated the sRNA population generated from the $5^{\prime}$ half of tRNA $^{\text {Val }}$. More specifically, of the 1,049,093 reads mapped to $\mathrm{tRNA}^{\mathrm{Val}}, 91 \%\left(954,675\right.$ reads) aligned to the $33^{\mathrm{rd}} \mathrm{nt}$ from the $5^{\prime}$ terminus of tRNA ${ }^{\text {Val }}$, and $7 \%(73,437$ reads) aligned to the $34^{\text {th }}$ nt from the $5^{\prime}$ end of the tRNA ${ }^{\text {Val }}$ molecule (Fig. 2c). Taken together, the data presented in Fig. $2 \mathrm{c}$ strongly suggested that in epididymal spermatozoa, the endonuclease responsible for tRF5 processing from tRNA substrates has a cleavage preference for the $5^{\prime}$ half of its tRNA substrates, and that this cleavage event results in the generation of tRF5 sRNAs, predominantly 31-33-nt in length.

\section{Experimental validation of the enrichment of a specific subpopulation of transfer RNA-derived RNA fragment small RNAs in cauda spermatozoa}

Profiling of the tRF5 landscape of epididymal spermatozoa clearly revealed that the abundance of the tRF5 ${ }^{\mathrm{Ala}}$, $\mathrm{tRF}^{\mathrm{Glu}}{ }^{\mathrm{t}} \mathrm{tRF}^{\mathrm{Gly}}$, tRF5 ${ }^{\mathrm{Leu}}, \mathrm{tRF}^{\mathrm{Lys}}$ and $\mathrm{tRF}^{\mathrm{Val}}$ sRNAs was enriched in spermatozoa sourced from the distal cauda segment of the mouse epididymis (Additional file 1: Table S4). Therefore, $\mathrm{tRF}^{\mathrm{Ala}}{ }^{\mathrm{A}} \mathrm{tRF} 5^{\mathrm{Glu}}, \mathrm{tRF} 5^{\mathrm{Gly}}, \mathrm{tRF} 5^{\mathrm{Leu}}, \mathrm{tRF} 5^{\mathrm{Lys}}$ and $\mathrm{tRF} 5^{\mathrm{Val}}$ abundance was quantified across the spermatozoa samples sourced from the caput, corpus and cauda segments of the mouse epididymis via the quantitative reverse transcriptase polymerase chain reaction (RT-qPCR) approach. RT-qPCR readily confirmed the sRNA sequencing identified trend of enriched tRF5 abundance in cauda sperm, compared to caput sperm, for the six tRF5 sRNAs assessed (Fig. 3). Although the degree of variation identified by the sRNA sequencing and RT-qPCR approaches differed, these two analyses did however reveal the matching trend of elevated tRF5 abundance in cauda spermatozoa compared to the abundance of this specific tRF5 subpopulation in sperm cells sampled from the proximal caput segment of the mouse epididymis (Fig. 3; Additional file 1: Table S6). In addition, RT-qPCR also confirmed the sRNA sequencing identified trend of elevated tRF5 $5^{\text {Ala }}$ (Fig. 3a), tRF5 $^{\text {Glu }}$ (Fig. 3b), tRF5 ${ }^{\text {Gly }}$ (Fig. 3c), tRF5 ${ }^{\text {Leu }}$ (Fig. 3d), tRF5 $5^{\text {Lys }}$ (Fig. 3e) and tRF5 ${ }^{\mathrm{Val}}$ (Fig. 3f) abundance in cauda spermatozoa, compared to the respective level of each of the six quantified sRNAs in spermatozoa sourced from the corpus epididymis (Additional file 1: Table S5). Therefore, when taken together, the data presented in Fig. 3 clearly confirms that tRF5 abundance was enriched in sperm sourced from the distal cauda epididymis segment compared to the level of this specific tRF5 subpopulation in spermatozoa sourced from either of the two more proximal segments of the mouse epididymis.

\section{ANGIOGENIN is the endonuclease likely responsible for the production of the cauda spermatozoa enriched transfer RNA-derived RNA fragment small RNA subpopulation}

Previous research has revealed the involvement of numerous endonucleases in the production of the various subpopulations of tRF sRNAs that accumulate in mammalian cells, including Dicer (DCR), RNase P, RNase Z and ELAC2 [31, 45, 54, 58, 60]. The endonuclease ANGIOGENIN (ANG) has also been demonstrated to play a role in tRF production, specifically; the production of tRNA halves [31, 38, 42, 62, 63]. Due to the exclusive mapping of the tRF5 sRNAs which belonged to the cauda spermatozoa enriched tRF5 subpopulation to the $5^{\prime}$ half of each mature tRNA from which they were processed, together with alignment of the $3^{\prime}$ terminal nucleotide of members of this sRNA subpopulation to immediately adjacent to the anticodon trinucleotide of the tRNA anticodon arm, ANG was identified as the endonuclease likely responsible for tRF5 production.

Considering that tRF5 accumulation was repeatedly demonstrated to be enriched in cauda sperm cells over spermatozoa sourced from the two more proximal 

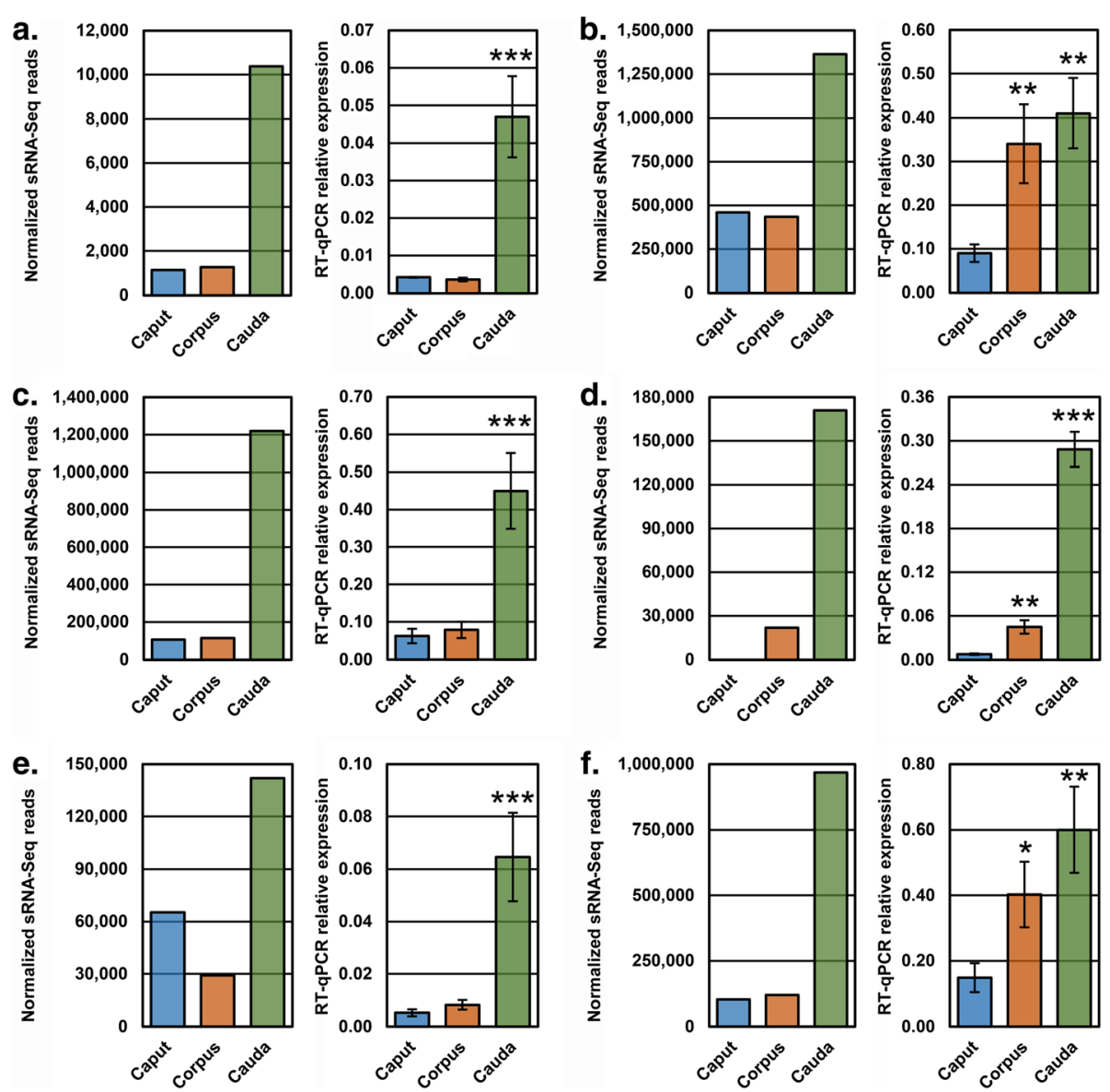

Fig. 3 Experimental validation of enriched tRNA-derived RNA fragment small RNA abundance in cauda spermatozoa. a to $\mathbf{f}$ The sRNA sequencing identified trend of enriched tRF5 abundance in cauda spermatozoa was experimentally validated via the use of the RT-qPCR approach for the

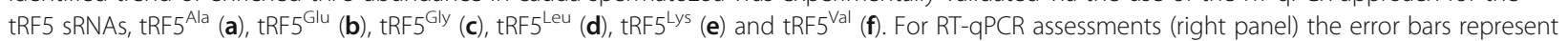
the standard error of the mean (SEM) of three biological replicates. Asterisks indicate significant difference $\left(p<0.05=* ; p<0.01={ }^{* *} ; p<\right.$ $\left.0.001{ }^{* * *}\right)$ compared to tRF5 sRNA abundance quantification in caput spermatozoa

epididymal segments, ANG abundance was assessed via a standard immunoblotting approach in spermatozoa sourced from the caput, corpus and cauda epididymis (Fig. 4). Immunoblotting readily revealed that the ANG endonuclease was highly abundant in caput sperm cells, lowly abundant in spermatozoa sourced from the corpus epididymis, and remained below the detection sensitivity level in cauda spermatozoa. Failure to detect ANG in sperm cells sourced from the cauda segment of the mouse epididymis, the segment of the tract in which sRNA sequencing and RT-qPCR revealed tRF5 accumulation to be highly enriched, may seem counterintuitive. However, previous research has demonstrated that sRNA molecules must be in an 'unbound' or 'free' state from either their effector protein machinery, or targeted nucleic acids, in order to facilitate their efficient detection by methods such as those employed here (i.e., RNA sequencing and RTqPCR) [64, 65]. Therefore, considering the ANG abundance data presented in Fig. 4, together with the tRF5 accumulation data presented in Fig. 3, our results strongly suggest that tRF5 sRNAs were readily detected in cauda spermatozoa due to much higher levels of 'unbound' or 'free' tRF5 sRNAs being harbored by the sperm cells sourced from the distal segment of the mouse epididymis in the absence of binding of this sRNA species by the tRFspecific endonuclease, ANG.

Profiling of the sRNA landscapes of our epithelium, epididymosome and spermatozoa specific datasets $[16$, $23,48-50]$ to determine the degree of extracellular communication between the epididymis soma and the transiting spermatozoa has previously revealed contrasting results. Namely, the abundance profiles of numerous miRNA sRNAs closely matched across the soma and spermatozoa samples sourced from the three epididymal segments, with similar trends in abundance for these miRNAs also documented in caput, corpus and cauda epididymosomes [16]. This suggested that members of this miRNA cohort are initially produced in the 


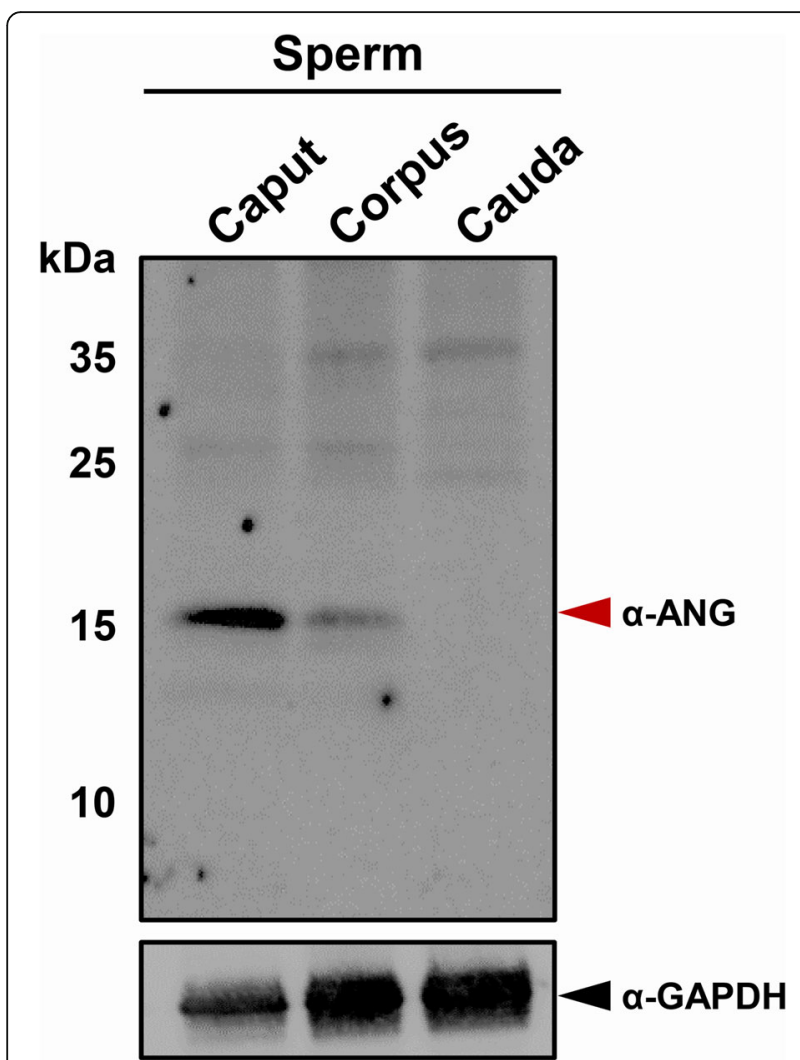

Fig. 4 Assessment of the abundance of the ANGIOGENIN endonuclease in caput, corpus and cauda spermatozoa. The abundance of the ANG endonuclease in spermatozoa sourced from the caput, corpus and cauda segments of the mouse epididymis was assessed via a standard immunoblotting approach. The specificity of the anti-ANG antibodies was confirmed via the inclusion of whole tissue lysates sampled from the mouse liver and anti-GAPDH (GLYCERALDEHYDE 3-PHOSPHATE DEHYDROGENASE) antibodies were used as the internal loading control. The red colored arrowhead indicates the immunoblot product of the expected size specific to the ANG endonuclease ( $15 \mathrm{kDa}$ ). The ANG and GAPDH antibodies were commercially sourced from suppliers Abcam (antibody ID: ab10600) and Sigma-Aldrich (product number: G9545), respectively

epithelial cells that line the tract, and post their production, these miRNAs are subsequently packaged into the epididymosomes that are secreted into the epididymal lumen to facilitate their delivery to the transiting spermatozoa. However, for the piRNA species of sRNA, abundance profiling failed to identify matching piRNA abundance trends for either the epithelial cells or epididymosomes isolated from the cauda segment of the epididymis, compared to sperm cells sampled from the same epididymal segment [23]. This initially formed an unexpected result to suggest that this sRNA species is produced in the sperm cells themselves, rather than being transferred to the transiting spermatozoa by epididymosomes post exosome production and packaging in the epididymis epithelial cells. We therefore next sought to determine whether the cauda spermatozoa enriched
tRF5 subpopulation was either; (1) produced in the soma, selectively packaged into epididymosomes, and then transferred to the spermatozoa via an epididymosome-mediated mode of delivery (as we have demonstrated previously for numerous miRNA sRNAs), or; (2) generated in the sperm cells themselves as the spermatozoa progress through their epididymal maturation (as we have demonstrated previously for a subset of piRNA sRNAs).

The tRF5 subpopulation enriched in cauda spermatozoa was determined to total 6,555,082 reads across the caput, corpus and cauda spermatozoa sequencing datasets (Additional file 1: Table S4). In comparison, only 716,268 and 1,330,172 sRNA reads specific to the cauda spermatozoa enriched tRF5 subpopulation were detected in the epithelial cell and epididymosome sequencing datasets, respectively (Additional file 1: Table S6). Compared to epididymal spermatozoa, the 9.2- and 4.9-fold reduction in the abundance of tRF5 sRNAs in soma and epididymosome samples respectively, revealed that although sRNA sequences specific to the cauda spermatozoa enriched tRF5 subpopulation were detected in epididymal epithelial cells and the extracellular vesicles that these cells secrete, they accumulated to considerably reduced degrees in both samples. Further evidence of enrichment of the tRF5 subpopulation in cauda spermatozoa is provided via comparison of the degree that this subpopulation represents of the total tRF population for each sample. In spermatozoa for example, the cauda enriched tRF5 subpopulation (6,555,082 reads) represented $71.8 \%$ of the $9,126,637$ reads mapped to tRF sRNAs (Additional file 1: Table S2). However, in the epididymal epithelial cell and epididymosome sequencing datasets, sRNA sequences aligning to the cauda spermatozoa enriched tRF5 subpopulation only represented 17.7 and $20.6 \%$ of the total tRF populations $(4,057,449$ and 6,457,554 tRF sRNA reads, respectively) of these respective samples (read data not shown).

The heatmap presented in Fig. 5 clearly shows the enriched abundance of the tRF5 subpopulation in cauda spermatozoa with the abundance of 14 out of the 15 tRF5 sRNAs accumulating to their highest degree in cauda spermatozoa (Additional file 1: Table S6). Only the tRF5 $^{\text {His }}$ sRNA failed to accumulate to its highest abundance level in cauda spermatozoa (Fig. 5) with higher $\mathrm{tRF}^{\mathrm{His}}$ read numbers detected in epididymosomes sampled from the caput, corpus and cauda segments of the mouse epididymis, and in sperm cells sourced from the caput and corpus epididymal segments (Additional file 1: Table S6). The analysis presented in Fig. 5 (Additional file 1: Table S6) also shows that the abundance of 11 out of the 15 sRNAs belonging to the cauda spermatozoa enriched tRF5 subpopulation was higher in the three epididymosome samples than in the 


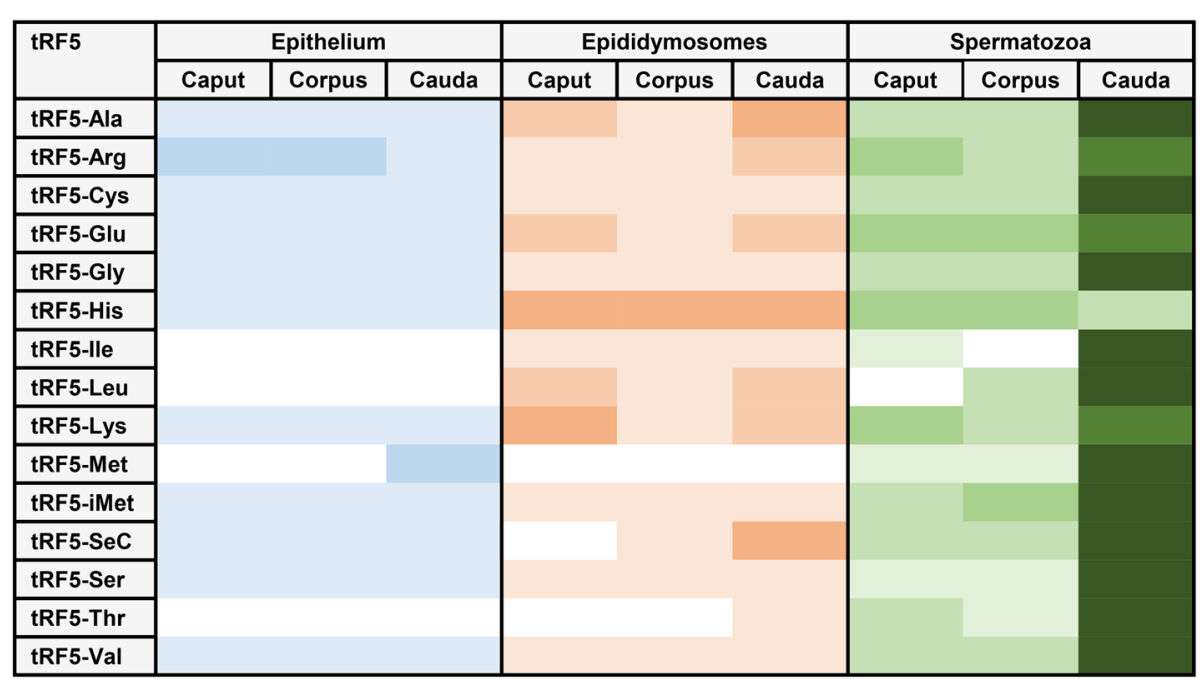

\begin{tabular}{|l|l|l|l|}
\hline Caput & Corpus & Cauda & $\%$ of total tRF Population \\
\hline & & & $0 \%$ (not detected) \\
& & 0.1 to $5.0 \%$ \\
\hline & & 5.1 to $10.0 \%$ \\
& & 10.1 to $25.0 \%$ \\
\hline & & & 25.1 to $50.0 \%$ \\
\hline & & 50.1 to $100.0 \%$ \\
\hline
\end{tabular}

Fig. 5 Heat map depicting the abundance of the cauda spermatozoa enriched tRNA-derived RNA fragment small RNA subpopulation in epithelial cells and epididymosomes sourced from the caput, corpus and cauda epididymis. To determine the degree (\%) that each assessed sample contributed to the total population of each of the 15 cauda spermatozoa enriched tRF5 sRNAs, the normalised read number for the epithelial cells, epididymosomes and spermatozoa samples sourced from the caput, corpus and cauda segments of the mouse epididymis was divided by the total read number of each tRF5 sRNA across all nine samples included in this analysis, namely the soma, epididymosomes and spermatozoa sourced from the caput, corpus and cauda epididymis

epithelium sample of each corresponding segment of the mouse epididymis: a finding that strongly suggests that the majority of the members of this tRF5 subpopulation are selectively packaged into epididymis extracellular vesicles in the soma that lines the tract prior to epididymosome secretion into the epididymal lumen. Furthermore, post epididymosome-mediated delivery of their tRF5 cargo to the transiting spermatozoa, the observed enrichment to the abundance of a specific subpopulation of tRF5 sRNAs, is likely due to the loss of ANG from the sperm cells harbored in the distal cauda segment of the mouse epididymis (Fig. 4), resulting in the ready detection of the now 'unbound' or 'free' tRF5 sRNAs in the cauda spermatozoa.

\section{Discussion}

In this study, we continue to build on our existing profiling of the global sRNA population of spermatozoa sourced from the caput, corpus and cauda segments of the epididymis of a physiologically normal mouse $[16,23$, 48-50]. Of central importance to the findings reported here was the identification of the sRNA species responsible for the dramatic change in the sRNA size distribution profile of spermatozoa sourced from the distal cauda segment of the mouse epididymis compared to the composition of the sRNA population of sperm isolated from the proximal caput epididymis. More specifically, the tRF species of sRNA was demonstrated to dominate the sRNA landscape of cauda spermatozoa, representing $64.7 \%$ of the 7,154,433 sRNA reads detected in cauda sperm (Fig. 1e). In direct contrast, miRNA sRNAs accounted for $49.8 \%$ of the global sRNA population $(8,287,165$ reads) of spermatozoa sourced from the proximal caput segment of the mouse epididymis (Fig. 1c).

Across numerous mouse cell or tissue types, miRNAs remain the most well characterised sRNA species owing to their repeated demonstration as central regulators of gene expression during standard growth and development [66-68], a regulatory role that miRNA sRNAs continue to direct when a cell is either exposed to stress $[69,70]$, or enters a diseased state [71, 72]. Considering the central role occupied by miRNA-directed gene expression regulation in development, our finding that miRNA abundance is reduced by 3.2 -fold in cauda sperm, compared to miRNA abundance in sperm cells sourced from the caput epididymis, is not surprising with the latter population of 
sperm cells having completed much of their developmental and functional maturation during its prolonged residence in the tract $[2,6]$.

We further show that the observed reduction to miRNA abundance in cauda sperm, compared with caput sperm, is largely accounted for by the reciprocal 2.7-fold enhancement in tRF sRNA abundance. Furthermore, much of the tRF enrichment in cauda sperm was the result of the elevated levels of tRFs of a specific size, namely tRFs of 30-33-nt in length (Fig. 2). Previous research across a diverse range of cells, tissues, organisms, and/or experimental conditions, has revealed that the tRF species of sRNA can be further categorized into functionally distinct subpopulations based on the size of the tRF molecule processed from the pre-tRNA/tRNA substrate, in combination with the position on the substrate from which the tRF sRNA is processed [42-44, $54,57,63]$. tRF molecules of longer length, specifically 30-35-nt tRFs, have been putatively assigned a role in mediating transgenerational heritance, potentially directing methylation status changes and/or chromatin structural modifications at the transcriptional level to regulate gene expression from the outset of embryo development [14, 19, 46, 55, 73-76]. Mediating such epigenetic modification is afforded to this tRF size class due to the additional demonstrations that members of this sRNA subpopulation are preferentially loaded by the cleavage incompetent $\mathrm{AGO}$ proteins, AGO1, AGO3 and AGO4 (and not by the cleavage competent AGO2) [42, 56,57 , and by the piRNA pathway machinery proteins, PIWI1, PIWI2 and PIWI4 [56, 63]: sRNA effector proteins demonstrated to function at the transcriptional level to regulate sRNA target sequence activity. Therefore, the enriched accumulation of 30-33-nt tRF5 sRNAs in spermatozoa sourced from the distal cauda epididymis suggests that this specifically sized tRF subpopulation could indeed form an important paternally derived 'epigenetic signal' passed on to the oocyte upon fertilization to regulate gene expression in early embryo development.

An extreme processing bias of the 15 tRNA molecules identified to act as substrate for the production of the cauda spermatozoa enriched tRF5 subpopulation was observed. More specifically, each member of the cauda spermatozoa enriched tRF subpopulation exclusively aligned to the $5^{\prime}$ half of the processed tRNA substrate to encompass the Dloop of the processed tRNA, and with a 3' terminal nucleotide that extended to almost immediately adjacent to the anticodon triplet of the anticodon arm of the tRNA: a finding that strongly indicated that the production of this tRF5 subpopulation was the result of the activity of a specific endonuclease (Fig. 2c). It is important to note here that a similar dramatic biasing in tRF processing (i.e., preferential processing of the $5^{\prime}$ half of mature tRNAs) and size distribution (i.e., 30-34-nt in length) has been reported previously for spermatozoa sourced from the mammalian epididymis $[14,17-20,47,74,77]$. Comparison of the data reported here to that documented in this series of previous findings indicates that the extreme tRF processing preference for the $5^{\prime}$ half of each tRNA substrate was not the result of an artefact, or a biasing, of the high throughput sequencing approach that was applied in this study to profile the sRNA landscape of epididymal spermatozoa.

A number of endonucleases have been previously associated with the production of the distinct subpopulations of tRF sRNAs that accumulate in mammalian cells, which together, represent the total tRF profile of the sample. For example, DCR, the RNase III-like endonuclease central to pre-miRNA processing for miRNA production [78-80], has been shown to also be required for the production specific tRF subtypes, namely; smaller sized tRFs of 19-22-nt in length, and which are predominantly processed from the 3 ' half of mature tRNAs [45, $54,58]$. However, these studies, and other reports [56, $60,61,63]$, also revealed that the production of tRFs of an equivalent size from other tRNA molecules is independent of DCR-catalyzed cleavage. When taken together, these contrasting findings suggest that only mature tRNA molecules with the ability to adopt secondary folding structures similar to those adopted by miRNA precursor transcripts, specifically the stem-loop folding structures of pre-miRNA transcripts, are recognized as a substrate by DCR for processing $[45,58]$. Interestingly, tRFs of a shorter length (19-22-nt), such as those that are the result of DCR-catalyzed tRNA processing appear to predominantly accumulate in the cell cytoplasm, whereas longer length tRF sRNAs (30-35-nt) that have originated from endonuclease processing of the $5^{\prime}$ side of the tRNA precursor, have been demonstrated to be imported back into the cell nucleus post production [56, 57, 63]. In addition, tRFs that accumulate in the cell cytoplasm, and which are of a similar size to miRNA sRNAs, have been associated with AGO2, the cleavage competent endonuclease central to miRNAdirected gene expression regulation in mammalian cells $[31,74]$. Again however, not all tRF sRNAs of this size class, and which accumulate in the cell cytoplasm, are loaded by AGO2 [31, 74]. This suggests that AGO2 loads sRNAs based on 'features' additional to size. Taken together, these findings indicate that this class of tRF sRNA acts in both an analogous and a distinct manner to the miRNA species of sRNA to modulate gene expression at the posttranscriptional level.

In addition to DCR, the endonuclease ELAC2 has also been demonstrated to play a role in the production stage of the tRF-directed RNA silencing pathway [44, 58,60]. Specifically, ELAC2 is required for the production of the $\mathrm{U}$ tRF3/tRF-1 subpopulation of tRF sRNAs via the 
processing of the variable number of uracil residues attached to the $3^{\prime}$ terminus of the cleaved tRNA precursor transcript $[44,58,60]$. In addition to ELAC2, the RNase $\mathrm{P}$ and RNase $\mathrm{Z}$ endonucleases have also been assigned functional roles in pre-tRNA/tRNA processing for production of either the $5^{\prime}$ or $3^{\prime}$ tRF subpopulations [31, $45,63]$. However, due to our failure to detect tRF sRNAs of origins that mapped to outside of the $5^{\prime}$ half of the 15 mature tRNAs determined to be used as processing substrates for the production of the cauda spermatozoa enriched tRF5 population, the ELAC2, RNase P and RNase $\mathrm{Z}$ endonucleases were considered to not play a functional role in the production of the cauda spermatozoa enriched tRF5 subpopulation detected in this study.

Alignment of the sRNAs to their respective tRNA precursors for all tRF5 sequences identified across the spermatozoa datasets for the three most abundant tRF5s in cauda sperm, including the tRF5 ${ }^{\text {Glu }}(2,258,567$ reads), tRF5 $^{\text {Gly }}\left(1,439,663\right.$ reads) and tRF5 ${ }^{\mathrm{Val}}$ (1,189,651 reads) sRNAs (Additional file 1: Table S4), revealed highly precise cleavage-based processing of the tRNA ${ }^{\text {Glu }}$, tRNA ${ }^{\text {Gly }}$ and tRNA ${ }^{\text {Val }}$ precursors, respectively (Fig. 2c). More specifically, three sRNAs of 31-, 32- and 33-nt in length dominated the $\mathrm{tRF} 5^{\mathrm{Glu}}$ and $\mathrm{tRF} 5^{\mathrm{Gly}}$ tRF populations processed from tRNA ${ }^{\text {Glu }}$ and tRNA ${ }^{\text {Gly }}$ precursors, and two tRF5 ${ }^{\mathrm{Val}}$ sRNAs (33- and 34-nt in length) were produced from the processing of the tRNA ${ }^{\text {Val }}$ precursor (Fig. 2c). Mapping of only 2 tRF sequences to tRNA ${ }^{\mathrm{Val}}$, compared to the alignment of three highly abundant tRF5 sRNAs to tRNA ${ }^{\text {Glu }}$ and tRNA ${ }^{\text {Gly }}$, strongly indicated that tRNA ${ }^{\mathrm{Val}}$ formed the preferred substrate for accurate endonuclease-mediated tRF5 sRNA processing in spermatozoa sourced from the mouse epididymis. The sRNAs, tRF5 ${ }^{\text {Glu }}$, tRF5 ${ }^{\text {Gly }}$ and tRF5 ${ }^{\text {Val }}$, have been previously demonstrated to accumulate to high levels in spermatozoa sourced from the proximal caput segment of the mouse epididymis, and for the levels of these three highly abundant tRFs to be further enriched in cauda spermatozoa [17]; a finding that closely aligns with the tRF profile constructed here. Cropley and colleagues [74] have also reported that the abundance of these three tRFs is enhanced in sperm extracted from obese males, compared to their accumulation levels in male mice of normal weight. The level of the tRF sRNA tRF5 ${ }^{\mathrm{Glu}}$, has also been demonstrated previously to be upregulated in the spermatozoa of male rats $\left(\mathrm{F}_{0}\right.$ animals $)$ and their offspring $\left(\mathrm{F}_{1}\right.$ animals) post paternal exposure to a high fat diet [46]. Documentation of highly similar tRF accumulation profiles across mice and rats readily highlights the potential functional importance of highly abundant tRF sRNAs in mammalian spermatozoa. Adding to this is the demonstration that the target genes of tRF5 ${ }^{\mathrm{Glu}}$ in the mouse play central roles in regulating transcription, alternate splicing, acetylation and phosphoprotein activation [18], molecular processes that are all involved in gene expression regulation. Furthermore, the expression of the target genes of the tRF $5^{\text {Gly }}$ sRNA were determined to be reduced in 2-cell stage embryos fertilized with spermatozoa from male mice fed a low protein diet, a diet that greatly enhanced the abundance of the tRF ${ }^{\text {Gly }}$ sRNA in the cauda sperm of these animals [19]. Taken together, these elegant findings readily highlight the importance of profiling the tRF landscape of the male reproductive tract of physiologically normal animals.

The predominance of 30-33-nt tRF5 sRNAs determined to originate from the $5^{\prime}$ half of each processed tRNA via a single cleavage event of the nucleotides immediately adjacent to the anticodon triplet of the anticodon arm, identified ANG as the endonuclease likely responsible for tRNA processing to produce the cauda spermatozoa enriched tRF5 subpopulation. ANG is an RNase A-like endonuclease that cleaves mature tRNAs into their $5^{\prime}$ and $3^{\prime}$ halves with the resulting sRNA subpopulations mapping to distinct cleavage positions on each processed tRNA precursor [38, 42, 59, 62, 63]. Further confidence that ANG was the endonuclease responsible for the production of the cauda spermatozoa enriched tRF5 subpopulation detected here was provided by the fact that none of the mapped sRNAs belonging to this subpopulation harbored sequences that aligned with either the leader or trailer sequences of any of the pretRNA molecules of the 15 tRNAs from which the mapped tRF sRNAs were determined to be processed from. The enriched abundance of a specific population of 30-33-nt tRF5s detected in our cauda spermatozoa sequencing dataset $[16,23,48-50]$, together with our complete failure to map any of the sequences of the sRNAs belonging to this specific tRF subpopulation to tRNA sequences outside of the $5^{\prime}$ and/or $3^{\prime}$ terminal residues of the respective mature tRNA substrates indicated that; (1) folded mature tRNAs formed the preferred substrate for ANG-catalyzed processing in mouse epididymal tissues, and; (2) the generated tRF5 sRNAs formed a distinct tRF subpopulation, and do not simply represent random degradation products of their highly abundant tRNA precursors.

Interestingly, tRNA halves form a distinct tRF subpopulation based on their longer length of 30-40-nt, compared to other tRF subpopulations which accumulate in other mammalian cell or tissue types, and which are of shorter length at 15-25-nt [18, 31, 43, 47, 57, 63]. Furthermore, longer length tRFs, such as tRNA halves, have been shown to accumulate in both the nuclear and cytoplasmic compartments of a cell, whereas shorter length tRFs (15-25-nt tRFs) appear to only accumulate in the cell cytoplasm [43, 45, 54, 63]. The observed enrichment in the abundance of 30-33-nt tRF5 sRNAs in cauda spermatozoa, could in part, be explained by the loss of 
tRF molecules of a shorter length, along with the majority of the remainder of the cytoplasmic contents of maturing spermatozoa when these cells shed their cytoplasmic droplets during their protracted descent through the caput, corpus and cauda segments of the epididymis. Adding to this theory is the demonstration that longer length tRF sRNA specifically accumulate in the head of the sperm [14, 47, 73], a position within the mature male gamete that would readily facilitate their transfer to the oocyte upon fertilization. It is important to note here that tRF sRNAs of longer length are predicted to preferentially target DNA-based sequences in either the promoter or $5^{\prime}$ untranslated region (UTR) of their target genes $[14,18,58,73,74]$ : a finding that indicates that tRFs, which are of a similar size to piRNA sRNAs, may also potentially direct their mechanism of gene expression regulation at the transcriptional level, and not at the posttranscriptional level, as demonstrated for the well characterized miRNA species of sRNA: a sRNA species that almost exclusively regulates target gene expression at the posttranscriptional level in the cytoplasm of mammalian cells.

Both the sRNA sequencing and RT-qPCR (Fig. 3) approaches used to profile the sRNA landscape of spermatozoa showed that the accumulation of a distinct subpopulation of tRF5 sRNAs was enriched in sperm cells sourced from the cauda segment of the mouse epididymis. This highly biased tRF accumulation profile repeatedly identified ANG as the endonuclease likely responsible for tRF5 production. Immunoblot assessment of ANG abundance in caput, corpus and cauda spermatozoa revealed an initially curious abundance trend, that is; ANG was highly abundant in spermatozoa sourced from the proximal caput segment, of low abundance in corpus sperm, and failed to accumulate to detectable levels in spermatozoa sourced from the distal cauda segment of the epididymis (Fig. 4). However, when taken together with the previous demonstrations that; (1) tRFs of longer length (30-40-nt) preferentially accumulate in the cell nucleus (the head of cauda spermatozoa) [14, 47], and; (2) sRNAs need to be completely free from their interacting molecules (proteins and/or other nucleic acids to which they are usually bound) to facilitate accurate determination of their abundance [64, 65], the loss of ANG, potentially via ANG being shed along with the other remnants of the sperm cell cytoplasm in the cytoplasmic droplet, may provide an explanation for the observed enrichment of a specific subpopulation of tRF sRNAs in cauda spermatozoa. The loss of ANG in cauda sperm may also indicate that removal of the endonuclease responsible for the production of this specific sRNA subpopulation is required in the male gamete to ensure that tRF sRNAs are in an 'unbound' or 'free' state in order to promote their loading by the tRF effector machinery proteins to direct their specific mechanism of target gene expression regulation upon oocyte fertilization.

\section{Conclusion}

Here we report on our continued mapping of the sRNA landscape of the spermatozoa sourced from a physiologically normal mouse epididymis. The tRF species of sRNA is the most abundant species of sRNA in cauda sperm, which is in direct contrast with spermatozoa sourced from the proximal caput epididymis where the miRNA sRNA species is the most abundant. In cauda sperm, the accumulation of a specific tRF5 subpopulation was enriched, specifically tRF sRNAs of a longer length, 30-33-nt, and which were exclusively derived from the $5^{\prime}$ half of each tRNA molecule used as a substrate for tRF sRNA processing. Furthermore, the RNase A-like endonuclease, ANG, was determined to be the endonuclease likely responsible for tRF sRNA production in mouse epididymal spermatozoa. We have now profiled the abundance trends of the three most thoroughly characterized sRNA species (the miRNA, piRNA and tRF sRNA species), in spermatozoa sourced from a physiologically normal mouse epididymis. Together, this work forms an important starting point for future studies to document alternations to the sRNA landscape of the mouse epididymis when the reproductive tract of the male mouse is exposed to various stressors. For cauda sperm specifically, 3033-nt tRF5 sRNAs form an ideal candidate to function as a 'epigenetic signal' in transgenerational studies that document the consequence of exposure of the paternal linage to stress.

\section{Methods}

\section{Ethics statement and animal care}

Each of the experimental protocols described here were conducted according to the University of Newcastle's Animal Care and Ethics Committee approval number A2013-322, and in accordance with the relevant national and international guidelines. All samples were exclusively extracted from Swiss mice that were housed under controlled conditions of $16 \mathrm{~h}(\mathrm{~h})$ of light and $8 \mathrm{~h}$ of dark and a constant temperature of $21-22^{\circ} \mathrm{C}$. Animals were supplied with food and water ad libitum and prior to sampling from 2-month-old male mice, animals were euthanised via treatment with $\mathrm{CO}_{2}$.

\section{Sampling of epididymal spermatozoa, epithelium and epididymosomes}

The epididymides were removed from 2-month-old male mice that had had their vasculature perfused with prewarmed phosphate buffered saline (PBS) to minimise the 
possibility of blood contamination immediately after these animals were euthanised. Post separation from any remaining fat or connective tissue, the extracted epididymides were dissected into the three anatomically distinct segments of the epididymis (caput, corpus and cauda segments) and spermatozoa from the cauda epididymis extracted via a method [23] distinct to that used for caput and corpus sperm extraction $[49,50]$. For collection of the samples used to obtain the sRNA sequencing data for the construction of the Fig. 5 heatmap, epithelial tissue was sampled as previously described $[49,50]$ using an approach adapted from the originally reported methodology [81]. Similarly, epididymosome fractions were obtained using our previously described protocols [16, 23].

\section{Total RNA extraction and sequencing of the small RNA fraction}

The Direct-zol RNA MiniPrep kit was used to extract total RNA from the caput, corpus and cauda epididymis sourced spermatozoa according to the manufacturer's protocol (Zymo Research Corporation, Australia). Post extraction, each total RNA preparation was treated with DNase (Promega, Australia) to remove any contaminating DNA and sample purity assessed using a spectrophotometer and the absorbance wavelengths, 260 and 280 $\mathrm{nm}$. Only samples with an $\mathrm{A}_{280} / \mathrm{A}_{260}$ absorbance ratio of greater than 1.8 were deemed to be of an appropriate level of quality to include in the subsequent sRNA sequencing experiments. Each biological replicate analysed by the sRNA sequencing approach was composed of preparations from at least nine animals and two such biological replicates were sequenced for analysis of the sRNA landscape of epididymal spermatozoa. All samples were prepared for high throughput sequencing of the sRNA fraction via the Illumina TruSeq small RNA sample preparation protocol according to the manufacturer's methods (Illumina, Australia) at the Melbourne node of the Australian Genome Research Facility (AGRF). As previously described [16, 23, 48-50], sRNA libraries were generated using an Illumina HiSeq-2000 RNA sequencing platform and 50 base-pair single end chemistry at the AGRF. The resulting sequencing libraries were processed and normalised according to our previously reported approach [23] with the exception that tRF sRNA abundance was normalised against the abundance of U6, a snRNA demonstrated to uniformly accumulate across the three segments of the mouse epididymis. Furthermore, each dataset can be publicly accessed via its deposition in the Gene Expression Omnibus (GEO) at the National Center for Biotechnology Information (NCBI) and the use of the GEO accession numbers, GSE70198 (spermatozoa), GSE70197 (epithelium), and GSE79500 (epididymosomes).
Quantitative reverse transcriptase polymerase chain reaction experimental validation of transfer RNA-derived RNA fragment small RNA enrichment in cauda spermatozoa

The sequencing identified enrichment of tRF5 sRNA abundance in cauda epididymis sourced spermatozoa was experimentally validated using a standard quantitative reverse transcriptase polymerase chain reaction (RT-qPCR) approach with custom (non-locked nucleic acid modified) Taqman sRNA assay reagents according to the manufacturer's instructions (Thermo Fisher Scientific, Australia). The adopted approach allows for the specific detection and amplification of the targeted tRF sRNA under assessment via the use of the primer sequences, $\mathrm{tRF} 5^{\mathrm{Ala}}\left(5^{\prime}\right.$ - GGGG ATGTAGCTCAGTG GTAGAG - $\left.3^{\prime}\right)$, tRF5 $5^{\text {Glu }}\left(5^{\prime}\right.$ - TCCC TGGTGGTCTAGTGGTTAGGATTCGG C - 3'), tRF5 Gly (5' - GCATTG GTGGTTCAGTGGTAGAATTCTCGC CT - 3'), tRF5 ${ }^{\text {Leu }}\left(5^{\prime}\right.$ - GGTAGCGTG GCCGAGCGGT CTAAGGC - 3'), tRF5 ${ }^{\text {Lys }}\left(5^{\prime}\right.$ - GCCCGGATAGCTCAGT CGGTAGAGCATCAGAC - $\left.3^{\prime}\right)$ and tRF5 $5^{\mathrm{Val}}\left(5^{\prime}-\right.$ GTTT CCGT AGTGTAGTGGTTATCACGTTCGCCTC - 3') for complementary DNA (cDNA) synthesis. A Light Cycler 96 SW 1.1 (Roche, Australia) cycling machine was used for the RT-qPCR-based quantification of the six tRFs assessed with the abundance of each quantified tRF5 sRNA normalised against the abundance of the U6 snRNA (snRNA; assay ID 001973; Thermo Fisher Scientific, Australia), a sRNA known to uniformly accumulate across the three anatomically distinct segments of the mouse epididymis. The quantification of tRF abundance was performed in biological triplicate and each biological replicate was composed of samples sourced from six to nine, 2-month-old male mice, which were separate from those animals used for sRNA sequencing.

\section{Immunoblot assessment of the abundance of the ANGIOGENIN endonuclease}

Total protein was extracted from spermatozoa sourced from the caput, corpus and cauda epididymal segments of 2-month-old male mice via boiling the extracts at $100{ }^{\circ} \mathrm{C}$ for $5 \mathrm{~min}$ in modified sodium dodecyl sulphate, polyacrylamide gel electrophoresis (SDS-PAGE) sample buffer (2\% $\mathrm{w} / \mathrm{v}$ SDS, $10 \% \mathrm{w} / \mathrm{v}$ sucrose in $0.1875 \mathrm{M}$ Tris, $\mathrm{pH} 6.8$, supplemented with protease inhibitor cocktail). Following the initial extraction, the insoluble protein fraction was removed via centrifugation at 20,000 $\mathrm{g}$ for $10 \mathrm{~min}$ at room temperature. Post centrifugation, the supernatant was used to perform protein estimations with a DC Protein Assay kit (Bio-Rad, Australia) according to the manufacturer's instructions. Based on these estimations, each sample was diluted to a working concentration of 15 micrograms $(\mu \mathrm{g})$ in modified SDS-PAGE buffer $(2 \% \mathrm{v} / \mathrm{v} \beta$ mercaptoethanol, $2 \% \mathrm{w} / \mathrm{v}$ SDS, $10 \% \mathrm{w} / \mathrm{v}$ sucrose in 0.1875 $\mathrm{M}$ Tris, $\mathrm{pH} 6.8$, supplemented with bromophenol blue) 
and then boiled at $100{ }^{\circ} \mathrm{C}$ for $5 \mathrm{~min}$. Denatured samples were immediately transferred to ice and incubated for 5 $\mathrm{min}$, and post this incubation period, samples were next resolved by SDS-PAGE and then transferred to a nitrocellulose membrane by electroblotting. Blotted membranes were blocked with $3 \% \mathrm{w} / \mathrm{v}$ bovine serum albumin (BSA; in Tris buffered saline (TBS), $\mathrm{pH} 7.4$ ) for $1 \mathrm{~h}$ at room temperature, before being probed overnight at $4{ }^{\circ} \mathrm{C}$ with anti-ANG (1:500 dilution) primary antibodies in TBS containing $1 \% \mathrm{w} / \mathrm{v}$ BSA and $0.1 \% \mathrm{v} / \mathrm{v}$ polyoxyethylene sorbitan monolaurate (Tween-20; TBST). The probed membrane was washed with three changes of fresh TBST, followed by incubation for $1 \mathrm{~h}$ at room temperature with the appropriate horseradish peroxidase-conjugated secondary antibody (1:1000 dilution) in 1\% w/v BSA in TBST. Membranes were washed three times with fresh changes of TBST and the labelled proteins visualized via the use of an enhanced chemiluminescence kit (GE Healthcare, Australia) and an ImageQuant LAS 4000 imager (Fujifilm, Australia). Each immunoblotting experiment was repeated three times with a representative blot presented here (Fig. 4). A parallel approach (as reported above for ANG) was used to probe for the housekeeping protein, GAPDH (1: 1000 dilution), to compare the total amount of protein loaded for each spermatozoa sample post stripping of the ANG-probed membrane. Furthermore, and post stripping of the GAPDH-probed membrane, a negative control was included in these analyses via repeating all blocking and probing steps exactly as outlined above except that the primary antibody was substituted with antibody buffer (secondary antibody only controls). The ANG and GAPD $\mathrm{H}$ antibodies were commercially sourced from suppliers Abcam (antibody ID: ab10600) and Sigma-Aldrich (product number: G9545), respectively.

\section{Supplementary Information}

The online version contains supplementary material available at https://doi. org/10.1186/s41544-020-00058-x.

Additional file 1: Table S1. Abundance of 19- to 36-nt small RNAs in epididymal spermatozoa, Table S2. Small RNA species abundance in spermatozoa sourced from the mouse epididymis, Table S3. Abundance of 27-35-nt tRFs in caput, corpus and cauda spermatozoa, Table S4. Abundance of cauda spermatozoa enriched tRF5 small RNAs, Table S5. RT-qPCR confirmation of tRF5 small RNA enrichment in cauda spermatozoa, Table S6. Abundance of the cauda spermatozoa enriched tRF5 small RNA population across epithelial cells, epididymosomes and spermatozoa sourced from the caput, corpus and cauda epididymis.

\section{Authors' contributions}

ALE and BN designed the study. $\mathrm{KH}, \mathrm{SJS}, \mathrm{ALE}$ and BN conducted the bioinformatic analysis. SJS, IRB and ALA collected the experimental material, isolated the RNA and protein fractions from the collected samples, and performed the RT-qPCR and immunoblotting experimental analyses. ALE, MDD and BN drafted the manuscript. SJS, IRB, ALA, KH, MDD, ALE and BN have read and approved the manuscript.

\section{Funding}

This research was supported by a National Health and Medical Research Council of Australia (NHMRC) Project Grant (APP1147932) awarded to BN and MDD. BN is the recipient of an NHMRC Senior Research Fellowship (APP1154837).

\section{Availability of data and materials}

The sRNA datasets relating to this study can be publicly accessed via the Gene Expression Omnibus (GEO) at the National Center for Biotechnology Information (NCBI) and the use of the GEO accession numbers, GSE70198 (spermatozoa), GSE70197 (epithelium), and GSE79500 (epididymosomes).

\section{Ethics approval and consent to participate}

Each of the experimental protocols described here were conducted according to the University of Newcastle's Animal Care and Ethics Committee approval number A-2013-322, and in accordance with the relevant national and international guidelines.

\section{Consent for publication}

Not applicable.

\section{Competing interests}

The authors declare that they have no competing interests.

\section{Author details}

${ }^{1}$ Priority Research Centre for Reproduction Science, School of Environmental and Life Sciences, The University of Newcastle, Callaghan, New South Wales 2308, Australia. ${ }^{2}$ Perth Children's Hospital, Nedlands, Western Australia 6009, Australia. ${ }^{3}$ Cancer Signalling Research Group, Medical Biochemistry, School of Biomedical Sciences and Pharmacy, Faculty of Health and Medicine, The University of Newcastle, Callaghan, New South Wales 2308, Australia. ${ }^{4}$ Priority Research Centre for Cancer Research, Innovation and Translation, Faculty of Health and Medicine, Hunter Medical Research Institute, New Lambton, New South Wales 2305, Australia.

Received: 3 April 2020 Accepted: 20 October 2020

Published online: 01 November 2020

\section{References}

1. Hermo L, Pelletier RM, Cyr DG, Smith CE. Surfing the wave, cycle, life history, and genes/proteins expressed by testicular germ cells. Part 4: intercellular bridges, mitochondria, nuclear envelope, apoptosis, ubiquitination, membrane/voltage-gated channels, methylation/acetylation, and transcription factors. Microsc Res Tech. 2010;73(4):364-408.

2. Cornwall GA. New insights into epididymal biology and function. Hum Reprod Update. 2009;15(2):213-27.

3. Biggiogera M. Chromatin arrangement in mouse sperm nuclei: an ultrastructural cytochemical study. Mol Reprod Dev. 1989;1(2):91-7.

4. Calvin HI, Bedford JM. Formation of disulphide bonds in the nucleus and accessory structures of mammalian spermatozoa during maturation in the epididymis. J Reprod Fertil Suppl. 1971;13:65-75.

5. Hermo L, Oliveira RL, Smith CE, Au CE, Bergeron JJM. Dark side of the epididymis: tails of sperm maturation. Andrology. 2019;7(5):566-80.

6. Zhou W, De luliis GN, Dun MD, Nixon B. Characteristics of the Epididymal Luminal Environment Responsible for Sperm Maturation and Storage. Front Endocrinol (Lausanne). 2018;9:59.

7. Dacheux JL, Gatti JL, Dacheux F. Contribution of epididymal secretory proteins for spermatozoa maturation. Microsc Res Tech. 2003;61(1):7-17.

8. Aitken RJ, Nixon B, Lin M, Koppers AJ, Lee YH, Baker MA. Proteomic changes in mammalian spermatozoa during epididymal maturation. Asian J Androl. 2007;9(4):554-64.

9. Dacheux JL, Belleannée C, Jones R, Labas V, Belghazi M, Guyonnet B, Druart X, Gatti JL, Dacheux F. Mammalian epididymal proteome. Mol Cell Endocrinol. 2009;306(1-2):45-50

10. Sullivan R, Saez F. Epididymosomes, prostasomes, and liposomes: their roles in mammalian male reproductive physiology. Reproduction. 2013;146(1):R21-35.

11. Sullivan R. Epididymosomes: a heterogeneous population of microvesicles with multiple functions in sperm maturation and storage. Asian J Androl. 2015;17(5):726-9.

12. Martin-DeLeon PA. Epididymal SPAM1 and its impact on sperm function. Mol Cell Endocrinol. 2006;250(1-2):114-21. 
13. Belleannée C, Calvo É, Caballero J, Sullivan R. Epididymosomes convey different repertoires of microRNAs throughout the bovine epididymis. Biol Reprod. 2013;89(2):30.

14. Chen Q, Yan M, Cao Z, Li X, Zhang Y, Shi J, Feng GH, Peng H, Zhang X, Zhang Y, Qian J, Duan E, Zhai Q, Zhou Q. Sperm tsRNAs contribute to intergenerational inheritance of an acquired metabolic disorder. Science. 2016;351(6271):397-400

15. Trigg NA, Eamens AL, Nixon B. The contribution of epididymosomes to the sperm small RNA profile. Reproduction. 2019. https://doi.org/10.1530/REP-18-0480.

16. Reilly JN, McLaughlin EA, Stanger SJ, Anderson AL, Hutcheon K, Church K, Mihalas BP, Tyagi S, Holt JE, Eamens AL, Nixon B. Characterisation of mouse epididymosomes reveals a complex profile of microRNAs and a potential mechanism for modification of the sperm epigenome. Sci Rep. 2016;6:31794.

17. Conine CC, Sun F, Song L, Rivera-Pérez JA, Rando OJ. Small RNAs gained during Epididymal transit of sperm are essential for embryonic development in mice. Dev Cell. 2018;46(4):470-80.

18. Rompala GR, Mounier A, Wolfe CM, Lin Q, Lefterov I, Homanics GE. Heavy chronic intermittent ethanol exposure alters small noncoding RNAs in mouse sperm and Epididymosomes. Front Genet. 2018;9:32.

19. Sharma U, Conine CC, Shea JM, Boskovic A, Derr AG, Bing XY, Belleannee C, Kucukural A, Serra RW, Sun F, Song L, Carone BR, Ricci EP, Li XZ, Fauquier L, Moore MJ, Sullivan R, Mello CC, Garber M, Rando OJ. Biogenesis and function of tRNA fragments during sperm maturation and fertilization in mammals. Science. 2016;351(6271):391-6.

20. Sharma U, Sun F, Conine CC, Reichholf B, Kukreja S, Herzog VA, Ameres SL, Rando OJ. Small RNAs are trafficked from the epididymis to developing mammalian sperm. Dev Cell. 2018;46(4):481-94.

21. Nixon B, De luliis GN, Hart HM, Zhou W, Mathe A, Bernstein IR, Anderson AL, Stanger SJ, Skerrett-Byrne DA, Jamaluddin MFB, Almazi JG, Bromfield EG, Larsen MR, Dun MD. Proteomic profiling of mouse Epididymosomes reveals their contributions to post-testicular sperm maturation. Mol Cell Proteomics. 2019;18(S1):S91-S108.

22. Zhou W, Stanger SJ, Anderson AL, Bernstein IR, De luliis GN, McCluskey A, McLaughlin EA, Dun MD, Nixon B. Mechanisms of tethering and cargo transfer during epididymosome-sperm interactions. BMC Biol. 2019;17(1):35.

23. Hutcheon K, McLaughlin EA, Stanger SJ, Bernstein IR, Dun MD, Eamens AL, Nixon B. Analysis of the small non-protein-coding RNA profile of mouse spermatozoa reveals specific enrichment of piRNAs within mature spermatozoa. RNA Biol. 2017;14(12):1776-90.

24. Johnston DS, Jelinsky SA, Bang HJ, DiCandeloro P, Wilson E, Kopf GS, Turner TT. The mouse epididymal transcriptome: transcriptional profiling of segmental gene expression in the epididymis. Biol Reprod. 2005;73(3):404-13.

25. Sipilä P, Björkgren I. Segment-specific regulation of epididymal gene expression. Reproduction. 2016;152(3):R91-9.

26. Nixon B, De luliis GN, Dun MD, Zhou W, Trigg NA, Eamens AL. Profiling of epididymal small non-protein-coding RNAs. Andrology. 2019;7(5):669-80.

27. Zhang J, Liu Q, Zhang W, Li J, Li Z, Tang Z, Li Y, Han C, Hall SH, Zhang Y. Comparative profiling of genes and miRNAs expressed in the newborn, young adult, and aged human epididymides. Acta Biochim Biophys Sin Shanghai. 2010;42:145-53.

28. Zhang YL, Zhang JS, Zhou YC, Zhao Y, Ni MJ. Identification of microRNAs and application of RNA interference for gene targeting in vivo in the rat epididymis. J Androl. 2011;32:587-91.

29. Ni MJ, Hu ZH, Liu Q, Liu MF, Lu MH, Zhang JS, Zhang L, Zhang YL. Identification and characterization of a novel non-coding RNA involved in sperm maturation. PLoS One. 2011;6:e26053.

30. Li Y, Wang HY, Wan FC, Liu FJ, Liu J, Zhang N, Jin SH, Li JY. Deep sequencing analysis of small non-coding RNAs reveals the diversity of microRNAs and piRNAs in the human epididymis. Gene. 2012;497:330-5.

31. Li Z, Ender C, Meister G, Moore PS, Chang Y, John B. Extensive terminal and asymmetric processing of small RNAs from rRNAs, snoRNAs, snRNAs, and tRNAs. Nucleic Acids Res. 2012:40(14):6787-99.

32. Björkgren I, Saastamoinen L, Krutskikh A, Huhtaniemi I, Poutanen M, Sipilä P. Dicer1 ablation in the mouse epididymis causes dedifferentiation of the epithelium and imbalance in sex steroid signaling. PLoS One. 2012;7(6):e38457.

33. Belleannée C, Calvo E, Thimon V, Cyr DG, Légaré C, Garneau L, Sullivan R. Role of microRNAs in controlling gene expression in different segments of the human epididymis. PLoS One. 2012;7(4):e34996.

34. Belleannée C, Thimon V, Sullivan R. Region-specific gene expression in the epididymis. Cell Tissue Res. 2012;349(3):717-31.
35. Sharma IM, Rappé MC, Addepalli B, Grabow WW, Zhuang Z, Abeysirigunawardena SC, Limbach PA, Jaeger L, Woodson SA. A metastable rRNA junction essential for bacterial 30 S biogenesis. Nucleic Acids Res. 2018; 46(10):5182-94.

36. Sanghai ZA, Miller L, Molloy KR, Barandun J, Hunziker M, Chaker-Margot M, Wang J, Chait BT, Klinge S. Modular assembly of the nucleolar pre-60S ribosomal subunit. Nature. 2018;556(7699):126-9.

37. Lackmann F, Belikov S, Burlacu E, Granneman S, Wieslander L. Maturation of the $90 \mathrm{~S}$ pre-ribosome requires Mrd1 dependent U3 snoRNA and 35 S prerRNA structural rearrangements. Nucleic Acids Res. 2018;46(7):3692-706.

38. Fu H, Feng J, Liu Q, Sun F, Tie Y, Zhu J, Xing R, Sun Z, Zheng X. Stress induces tRNA cleavage by angiogenin in mammalian cells. FEBS Lett. 2009;583(2):437-42.

39. Kuhn CD. RNA versatility governs tRNA function: why tRNA flexibility is essential beyond the translation cycle. Bioessays. 2016;38(5):465-73.

40. Ishii TM, Kotlova N, Tapsoba F, Steinberg SV. The long D-stem of the selenocysteine tRNA provides resilience at the expense of maximal function. J Biol Chem. 2013;288(19):13337-44

41. Giegé R, Jühling F, Pütz J, Stadler P, Sauter C, Florentz C. Structure of transfer RNAs: similarity and variability. Wiley Interdiscip Rev RNA. 2012;3(1):37-61.

42. Keam SP, Hutvagner G. tRNA-derived fragments (tRFs): emerging new roles for an ancient RNA in the regulation of gene expression. Life (Basel). 2015; 5(4):1638-51.

43. Kumar P, Anaya J, Mudunuri SB, Dutta A. Meta-analysis of tRNA derived RNA fragments reveals that they are evolutionarily conserved and associate with AGO proteins to recognize specific RNA targets. BMC Biol. 2014;12:78.

44. Lee YS, Shibata Y, Malhotra A, Dutta A. A novel class of small RNAs: tRNAderived RNA fragments (tRFs). Genes Dev. 2009;23(22):2639-49.

45. Soares AR, Fernandes N, Reverendo M, Araújo HR, Oliveira JL, Moura GM, Santos MA. Conserved and highly expressed tRNA derived fragments in zebrafish. BMC Mol Biol. 2015;16:22.

46. de Castro BT, Ingerslev LR, Alm PS, Versteyhe S, Massart J, Rasmussen M, Donkin I, Sjögren R, Mudry JM, Vetterli L, Gupta S, Krook A, Zierath JR, Barrès $R$. High-fat diet reprograms the epigenome of rat spermatozoa and transgenerationally affects metabolism of the offspring. Mol Metab. 2015; 5(3):184-97.

47. Peng $H$, Shi J, Zhang Y, Zhang H, Liao S, Li W, Lei L, Han C, Ning L, Cao Y, Zhou Q, Chen Q, Duan E. A novel class of tRNA-derived small RNAs extremely enriched in mature mouse sperm. Cell Res. 2012;22(11):1609-12.

48. Anderson AL, Stanger SJ, Mihalas BP, Tyagi S, Holt JE, McLaughlin EA, Nixon B. Assessment of microRNA expression in mouse epididymal epithelial cells and spermatozoa by next generation sequencing. Genom Data. 2015;6:208-11.

49. Nixon B, Stanger SJ, Mihalas BP, Reilly JN, Anderson AL, Dun MD, Tyagi S, Holt JE, McLaughlin EA. Next generation sequencing analysis reveals segmental patterns of microRNA expression in mouse Epididymal epithelial cells. PLoS One. 2015;10(8):e0135605.

50. Nixon B, Stanger SJ, Mihalas BP, Reilly JN, Anderson AL, Tyagi S, Holt JE, McLaughlin EA. The microRNA signature of mouse spermatozoa is substantially modified during epididymal maturation. Biol Reprod. 2015;93(4):91.

51. Aravin AA, Sachidanandam R, Bourc'his D, Schaefer C, Pezic D, Toth KF, Bestor T, Hannon GJ. A piRNA pathway primed by individual transposons is linked to de novo DNA methylation in mice. Mol Cell. 2008;31(6):785-99.

52. Kim VN. Small RNAs just got bigger: Piwi-interacting RNAs (piRNAs) in mammalian testes. Genes Dev. 2006:20(15):1993-7.

53. Vourekas A, Zheng Q, Alexiou P, Maragkakis M, Kirino Y, Gregory BD, Mourelatos Z. Mili and Miwi target RNA repertoire reveals piRNA biogenesis and function of Miwi in spermiogenesis. Nat Struct Mol Biol. 2012;19(8):773-81.

54. Cole C, Sobala A, Lu C, Thatcher SR, Bowman A, Brown JW, Green PJ, Barton GJ, Hutvagner G. Filtering of deep sequencing data reveals the existence of abundant dicer-dependent small RNAs derived from tRNAs. RNA. 2009; 15(12):2147-60

55. Donkin I, Barrès R. Sperm epigenetics and influence of environmental factors. Mol Metab. 2018;14:1-11.

56. Keam SP, Young PE, McCorkindale AL, Dang TH, Clancy JL, Humphreys DT, Preiss T, Hutvagner G, Martin DI, Cropley JE, Suter CM. The human Piwi protein Hiwi2 associates with tRNA-derived piRNAs in somatic cells. Nucleic Acids Res. 2014:42(14):8984-95.

57. Kumar P, Mudunuri SB, Anaya J, Dutta A. tRFdb: a database for transfer RNA fragments. Nucleic Acids Res. 2015;43:D141-5.

58. Soares AR, Santos M. Discovery and function of transfer RNA-derived fragments and their role in disease. Wiley Interdiscip Rev RNA. 2017;8(5). https://doi.org/10.1002/wrna.1423. 
59. Yang XL, Schimmel P. Functional expansion of the tRNA world under stress. Mol Cell. 2011;43(4):500-2.

60. Wang Q, Lee I, Ren J, Ajay SS, Lee YS, Bao X. Identification and functional characterization of tRNA-derived RNA fragments (tRFs) in respiratory syncytial virus infection. Mol Ther. 2013;21(2):368-79.

61. Schorn AJ, Gutbrod MJ, LeBlanc C, Martienssen R. LTR-Retrotransposon control by tRNA-derived small RNAs. Cell. 2017;170(1):61-71.

62. Li Q, Hu B, Hu GW, Chen CY, Niu X, Liu J, Zhou SM, Zhang CQ, Wang Y, Deng ZF. tRNA-derived small non-coding RNAs in response to ischemia inhibit angiogenesis. Sci Rep. 2016;6:20850.

63. Kumar P, Kuscu C, Dutta A. Biogenesis and function of transfer RNA-related fragments (tRFs). Trends Biochem Sci. 2016;41(8):679-89.

64. Smith NA, Eamens AL, Wang MB. The presence of high-molecularweight viral RNAs interferes with the detection of viral small RNAs. RNA. 2010;16(5):1062-7.

65. Smith NA, Eamens AL. Isolation and detection of small RNAs from plant tissues. Methods Mol Biol. 2012;894:155-72.

66. Morita S, Horii T, Kimura M, Goto Y, Ochiya T, Hatada I. One Argonaute family member, Eif2c2 (Ago2), is essential for development and appears not to be involved in DNA methylation. Genomics. 2007;89(6):687-96.

67. Yu Z, Raabe T, Hecht NB. MicroRNA Mirn122a reduces expression of the posttranscriptionally regulated germ cell transition protein 2 (Tnp2) messenger RNA (mRNA) by mRNA cleavage. Biol Reprod. 2005;73(3):427-33.

68. Tang F, Kaneda M, O'Carroll D, Hajkova P, Barton SC, Sun YA, Lee C, Tarakhovsky A, Lao K, Surani MA. Maternal microRNAs are essential for mouse zygotic development. Genes Dev. 2007;21(6):644-8.

69. Aten S, Page CE, Kalidindi A, Wheaton K, Niraula A, Godbout JP, Hoyt KR, Obrietan K. miR-132/212 is induced by stress and its dysregulation triggers anxiety-related behavior. Neuropharmacology. 2019;144:256-70.

70. Stenz L, Schechter DS, Serpa SR, Paoloni-Giacobino A. Intergenerational transmission of DNA methylation signatures associated with early life stress. Curr Genomics. 2018;19(8):665-75.

71. Otsuka M, Zheng M, Hayashi M, Lee JD, Yoshino O, Lin S, Han J. Impaired microRNA processing causes corpus luteum insufficiency and infertility in mice. J Clin Invest. 2008:118(5):1944-54.

72. Gottardo F, Liu CG, Ferracin M, Calin GA, Fassan M, Bassi P, Sevignani C, Byrne D, Negrini M, Pagano F, Gomella LG, Croce CM, Baffa R. Micro-RNA profiling in kidney and bladder cancers. Urol Oncol. 2007;25(5):387-92.

73. Chen Q, Yan W, Duan E. Epigenetic inheritance of acquired traits through sperm RNAs and sperm RNA modifications. Nat Rev Genet. 2016;17(12):733-43.

74. Cropley JE, Eaton SA, Aiken A, Young PE, Giannoulatou E, Ho JWK, Buckland ME, Keam SP, Hutvagner G, Humphreys DT, Langley KG, Henstridge DC, Martin DIK, Febbraio MA, Suter CM. Male-lineage transmission of an acquired metabolic phenotype induced by grand-paternal obesity. Mol Metab. 2016;5(8):699-708.

75. Isganaitis E, Suehiro H, Cardona C. Who's your daddy?: paternal inheritance of metabolic disease risk. Curr Opin Endocrinol Diabetes Obes. 2017;24(1):47-55.

76. Rompala GR, Homanics GE. Intergenerational effects of alcohol: a review of paternal preconception ethanol exposure studies and epigenetic mechanisms in the male Germline. Alcohol Clin Exp Res. 2019:43(6):1032-45.

77. Gapp K, Jawaid A, Sarkies P, Bohacek J, Pelczar P, Prados J, Farinelli L, Miska E, Mansuy IM. Implication of sperm RNAs in transgenerational inheritance of the effects of early trauma in mice. Nat Neurosci. 2014;17(5):667-9.

78. Zhou L, Seo KH, He HZ, Pacholczyk R, Meng DM, Li CG, Xu J, She JX, Dong Z, Mi QS. Tie2cre-induced inactivation of the miRNA-processing enzyme dicer disrupts invariant NKT cell development. Proc Natl Acad Sci U S A. 2009;106(25):10266-71.

79. Seo KH, Zhou L, Meng D, Xu J, Dong Z, Mi QS. Loss of microRNAs in thymus perturbs invariant NKT cell development and function. Cell Mol Immunol. 2010;7(6):447-53.

80. Nagalakshmi VK, Ren Q, Pugh MM, Valerius MT, McMahon AP, Yu J. Dice regulates the development of nephrogenic and ureteric compartments in the mammalian kidney. Kidney Int. 2011;79(3):317-30.

81. Zuo WL, Li S, Huang JH, Yang DL, Zhang G, Chen SL, Ruan YC, Ye KN, Cheng $\mathrm{CH}$, Zhou WL. Sodium coupled bicarbonate influx regulates intracellular and apical pH in cultured rat caput epididymal epithelium. PLoS One. 2011;6(8):e22283.

\section{Publisher's Note}

Springer Nature remains neutral with regard to jurisdictional claims in published maps and institutional affiliations.

Ready to submit your research? Choose BMC and benefit from:

- fast, convenient online submission

- thorough peer review by experienced researchers in your field

- rapid publication on acceptance

- support for research data, including large and complex data types

- gold Open Access which fosters wider collaboration and increased citations

- maximum visibility for your research: over $100 \mathrm{M}$ website views per year

At BMC, research is always in progress.

Learn more biomedcentral.com/submissions 